\title{
The application of a vacuum-ultraviolet Fourier transform spectrometer and synchrotron-radiation source to measurements of bands of NO. VII. The final report
}

\author{
K. Yoshino ${ }^{\text {a) }}$ \\ Harvard-Smithsonian Center for Astrophysics, Cambridge, Massachusetts 02138
}

A. P. Thorne and J. E. Murray

Blackett Laboratory, Imperial College, London, SW7-2BZ, United Kingdom

A. S.-C. Cheung and A. L. Wong

Department of Chemistry, The University of Hong Kong, Hong Kong

T. Imajo

Japan Women's University, Tokyo 112-8681, Japan

(Received 28 September 2005; accepted 25 October 2005; published online 7 February 2006)

\begin{abstract}
Photoabsorption measurements of NO bands have been made by vacuum-ultraviolet Fourier transform spectrometry with a resolution of $0.12 \mathrm{~cm}^{-1}$ in the wavelength region of 166.2-196.2 nm. Accurate line positions are obtained for the $\delta(v, 0)$ bands with $v=2,3$, the $\epsilon(v, 0)$ bands with $v$ $=2,3$, and the $\beta(v, 0)$ bands with $v=10,12,14$. Absolute term values are found for the corresponding upper levels $C(2,3), D(2,3)$, and $B(10,12,14)$. Accurate rotational line integrated cross sections have also been obtained for the lines in these bands. Integrated cross sections reported in our earlier papers [J. Chem. Phys. 109, 1751 (1998); 112, 2251 (2000); 115, 3719 (2001); 116, 155 (2002); 117, 10621 (2002); 119, 8373 (2003)] have been revised, and the results reported here comprise the $\delta(v, 0)$ bands with $v=0-3$, the $\epsilon(v, 0)$ bands with $v=0-3$, the $\beta(v, 0)$ bands with $v=6,7,9-12,14$, and the $\gamma(3,0)$ band. For each band, the band oscillator strength is obtained from the sum of the line strengths of all rotational lines, and these are compared with other published values. (C) 2006 American Institute of Physics. [DOI: 10.1063/1.2138029]
\end{abstract}

\section{INTRODUCTION}

In a series of papers over the last few years we have reported measurements of accurate line positions and intensities of various bands: the $\gamma$ band $\left[A^{2} \Sigma^{+}-X^{2} \Pi_{r}\right]$, the $\beta$ band $\left[B^{2} \Pi_{r}-X^{2} \Pi_{r}\right]$, the $\delta$ band $\left[C^{2} \Pi_{r}-X^{2} \Pi_{r}\right]$, and the $\epsilon$ band $\left[D^{2} \Sigma^{+}-X^{2} \Pi_{r}\right]$ of NO between 180 and $198 \mathrm{~nm}$, measured in absorption with a vacuum-ultraviolet Fourier transform spectrometer. ${ }^{1-6}$ These measurements are needed for reliable modeling of the photodestruction of NO by solar radiation in the middle atmosphere. The absorption lines are narrow, and the necessity for high-resolution measurements to yield true cross sections has been discussed in our earlier papers. ${ }^{1-3} \mathrm{To}$ achieve the necessary resolution we combined vacuumultraviolet (vuv) Fourier transform spectrometry (FTS) with synchrotron radiation by taking the Imperial College (IC) vuv FT spectrometer to the synchrotron-radiation source at Photon Factory (PF), KEK, Japan, where a suitable zero dispersion two-grating predisperser is available on beamline 12-B. [The predisperser is necessary to limit the bandwidth to a few nanometers in order to achieve acceptable signal-tonoise $(\mathrm{S} / \mathrm{N})$ ratios.] We have used this combination of facilities to make ultrahigh-resolution cross-section measurements of NO in the wavelength region of 160-195 nm.

\footnotetext{
a) Author to whom correspondence should be addressed. Electronic mail: kyoshino@cfa.harvard.edu
}

Our six earlier papers ${ }^{1-6}$ give line wave numbers, integrated cross sections, and band oscillator strengths for the $\delta(1,0), \epsilon(0,0), \epsilon(1,0), \beta(6,0), \beta(9,0), \beta(11,0)$, and $\gamma(3,0)$ bands together with term values and molecular constants for the corresponding upper levels: $C(1), D(0,1), B(6,9,11)$, and $A(3)$. An additional paper ${ }^{7}$ discussed the absolute wavenumber calibration of the FT spectra and made some small corrections (less than $0.1 \mathrm{~cm}^{-1}$ ) to the line positions and term values of some of the bands. Prior to the work at PF, we also made measurements by FTS of the $\delta(0,0)$ and $\beta(7,0)$ bands, using a hydrogen discharge source as the background continuum; ${ }^{8}$ these yielded accurate line positions, together with term values for $C(0)$ and $B(7)$, but larger uncertainties in line intensities owing to low signal-to-noise ratio and uncertain column density measurements.

Herzberg et al. ${ }^{9}$ have made a rotational analysis of the $\delta$ and $\beta$ bands without publishing the observed line list. They pointed out the interaction of the $C(2)$ and $B(12)$ levels and also the $C(3)$ and $B(15)$ levels, the band origins of which are shifted in opposite directions by about 80 and $200 \mathrm{~cm}^{-1}$, respectively. Higher levels are almost completely mixed. Lagerqvist and Miescher ${ }^{10}$ presented the entire line lists of the $\delta$ and $\beta$ bands, and discussed the interaction between the $C^{2} \Pi$ and $B^{2} \Pi$ states. They mentioned that the lower $J$ levels of the $C(2)$ level are perturbed by the $B(12)$ level, and the high $J$ levels by the $B(13)$ level. Gallusser and Dressler ${ }^{11}$ studied theoretically the homogeneous perturbation of the ${ }^{2} \Pi$ state of 
$\mathrm{NO}$, and presented the perturbed and unperturbed band origins, the rotational constants, and the band oscillator strengths for the $\delta$ and $\beta$ bands. Raoult ${ }^{12}$ made a more complete study of the perturbation of ${ }^{2} \Pi-{ }^{2} \Pi$ Rydberg-valance states by using generalized quantum-defect theory. Recently Braun et al. ${ }^{13}$ observed the $E^{2} \Sigma^{+}-C^{2} \Pi$ bands $(\Delta v=0)$ in emission with a high-resolution Fourier transform spectrometer in the infrared region and tabulated the term values of the $A, D, E$, and $C$ states for $v=0-2$. Their values are compared with those from our previous papers in Ref. 7.

The cross-section measurements of NO bands by Bethke $^{14}$ were performed at low resolution, $0.04 \mathrm{~nm}$, in the presence of high-pressure Ar. The addition of the argon buffer gas ensured that the rotational lines were broadened beyond the instrumental resolution, allowing the true cross section to be presented. Guest and Lee ${ }^{15}$ used a synchrotron source and resolution of $0.03 \mathrm{~nm}$ for the cross-section measurements at very low pressure of NO gas, $2 \times 10^{-4}-7$ $\times 10^{-2}$ Torr. Chan et al. ${ }^{16}$ used the high-resolution dipole $(e, e)$ technique, which is not sensitive to the instrumental resolution. Luque and Crosley ${ }^{17}$ calculated the band oscillator strengths of the $A-X$ and $D-X$ systems. Very recently, Mayor et al. ${ }^{18}$ calculated the integrated cross section of rotational line of the $\delta(0,0)$ band of $\mathrm{NO}$ with the molecular quantum-defect orbital methology, and they compared their results with our previous results. ${ }^{8}$

In this paper we report the results of analysis of all the other bands observed with the synchrotron-FTS combination: $\delta(2,0), \delta(3,0), \beta(10,0), \beta(12,0), \beta(14,0), \epsilon(2,0)$, and $\epsilon(3,0)$, together with new measurements of $\delta(0,0)$ and $\beta(7,0)$. The uncertainty in the absolute wave numbers and term values is $0.03 \mathrm{~cm}^{-1}$ after calibration. ${ }^{7}$ The band oscillator strengths have been determined from line-by-line measurements because the spectral resolution is comparable with the Doppler widths of the lines.

\section{EXPERIMENT}

Details of the experimental procedures for recording high-resolution FT spectra of NO between 160 and $198 \mathrm{~nm}$ have been described in our earlier publications. ${ }^{1,2}$ Only a brief description of the experimental conditions will be given here. The optical path length and the pressure of NO gas depend on the particular band. The column densities of NO in these experiments were $8.32 \times 10^{15} \mathrm{~mol} \mathrm{~cm}^{-2}$ for the $\delta(0,0)$ and $\beta(7,0)$ bands, $9.24 \times 10^{15} \mathrm{~mol} \mathrm{~cm}^{-2}$ for the $\delta(2,0)$ and $\beta(12,0)$ bands, $1.47 \times 10^{16} \mathrm{~mol} \mathrm{~cm}^{-2}$ for the $\epsilon(2,0)$ band, $2.06 \times 10^{16} \mathrm{~mol} \mathrm{~cm}^{-2}$ for the $\delta(3,0)$ band, $3.70 \times 10^{16} \mathrm{~mol} \mathrm{~cm}^{-2}$ for the $\epsilon(3,0)$ band, 2.10 $\times 10^{17} \mathrm{~mol} \mathrm{~cm}^{-2}$ for the $\beta(14,0)$ band, and 2.22 $\times 10^{17} \mathrm{~mol} \mathrm{~cm}^{-2}$ for the $\beta(10,0)$ band. The signal-to-noise ratios in the continuum background were 34 to 70 depending on the wave numbers.

The transmission spectra were converted to optical depth by taking the logarithms of the intensity and fitting a smooth continuum to the regions between the lines. The absorption lines were fitted to Voigt profiles using the spectral reduction routine GREMLIN. ${ }^{19}$ Line parameters such as line position, linewidth, and integrated intensity of individual lines were determined through a nonlinear least-squares iterative procedure. The Voigt profile for the NO lines should be a convolution of a Gaussian due to the Doppler broadening and a Lorentzian arising from predissociation. In this case the Gaussian component of the best-fit Voigt function had a full width at half maximum (FWHM) of $0.175 \mathrm{~cm}^{-1}$ which is significantly larger than the value of $0.12 \mathrm{~cm}^{-1}$ expected for the Doppler width in our experimental conditions. This anomalous Gaussian width is considered to be due to drifts in alignment causing very small wave-number shifts over the long observation periods. ${ }^{20}$

As discussed in the previous paper ${ }^{7}$ on absolute wavenumber calibration, the FTS scaling factor can be treated as a constant shift over the limited wave-number range of a single band. A directly measured shift of $+0.087 \mathrm{~cm}^{-1}$ has been applied to the $\beta(10,0)$ band and a shift of $+0.083 \mathrm{~cm}^{-1}$ (the mean value of all the directly measured shifts) to all the other bands. The uncertainty in the absolute wave numbers is $0.03 \mathrm{~cm}^{-1}$, while the relative uncertainty is $0.01 \mathrm{~cm}^{-1}$ for the strongest lines.

As pointed out by Hudson and Carter, ${ }^{21}$ most crosssection measurements for molecular bands with fine rotational structures are severely distorted by the instrumental bandwidths. Plots of optical depth versus column density are never linear unless the spectral features are very much broader than the instrumental widths. All measured cross sections are weighted averages over the instrumental widths, so that the measured peak cross sections of the lines are lower limits to the true values and the wing cross sections are the upper limits. On the other hand, the integrated cross sections are much less sensitive to the instrumental width, and for the lines of small optical depth ("optically thin" lines) they are independent of instrumental width. We discussed these effects in more detail in the Appendix of Stark et al. ${ }^{22}$ In the present measurements we set the resolution of the FT spectrometer to $0.06 \mathrm{~cm}^{-1}$, about half of the Doppler width, to avoid the effects of the instrumental function, since the ratio of measured to true integrated cross sections for a line of optical depth 1.5 should then be 0.98 , within the experimental uncertainty. However, as explained above, the resolution is degraded to around $0.12 \mathrm{~cm}^{-1}$ by the alignment drift, and the effect on the integrated cross sections of the stronger lines becomes significant. We calculated the size of the effect for a Gaussian instrumental function of width $0.12 \mathrm{~cm}^{-1}$, equal to the Doppler width. (The FTS sinc instrument function convolved with a rectangular shift function of appropriate width can be closely approximated by a Gaussian.) The ratio of measured to true cross section varies from 0.93 at a measured optical depth of 0.5 to 0.77 at a measured optical depth of 1.5. We have applied an optical path-dependent correction based on this calculation to each observed line.

\section{RESULTS AND DISCUSSION}

\section{A. Line positions and term values}

The $\delta(0,0)$ and $\beta(7,0)$ bands in the wavelength region of 190-192 nm were recorded in our earlier work with the FT spectrometer, ${ }^{8}$ but we repeated the measurements of these bands for consistency of the series. We found complete 
agreement in line position measurements between the previous $^{8}$ and present work, with average differences of $0.001 \pm 0.017 \mathrm{~cm}^{-1}$, so we do not repeat the line positions and term values in this paper.

The absolute line wave numbers of the $\beta$ bands $(10,0)$, $(12,0)$, and $(14,0)$; the $\delta$ bands $(2,0)$ and $(3,0)$; and the $\epsilon$ bands $(2,0)$ and $(3,0)$ are presented in Table I. The $\beta(10,0)$ band is observed in the wavelength region of 181.5-182.7 $\mathrm{nm}$, in the shoulder of the strong $\delta(1,0)$ band, and the line positions are given in Table Ia. The upper levels of the $C(1)$ and $B(10)$ levels are perturbed around $J=13$, and the lines with $J>13$ are the tails of the $\delta(1,0)$ band. However, the bands appear as two separate bands and are presented in this way. The high column density used in these measurements allowed us to assign ten additional weak lines belonging to the $\delta(1,0)$ band, $^{2}$ and these are also presented in Table Ia. The $\delta(2,0)$ and $\beta(12,0)$ bands are observed in the wavelength region of 174.9-177.1 nm. Both bands appear with similar intensity, and their upper levels, $C(2)$ and $B(12)$, perturb each other strongly. The high $J$ of the $C(2)$ level are also perturbed by the $B(13)$ levels. ${ }^{23}$ A $\Lambda$-type doubling is observed for the $\delta(2,0)$ band, except for the low $J$ lines, but not for the $\beta(12,0)$ band. These results are presented in Tables Ib and Ic for the $\beta(12,0)$ and $\delta(2,0)$ bands, respectively. The $\epsilon(2,0)$ and $\beta(13,0)$ bands are observed in the wavelength regions of $172.3-173.5$ and $170.7-172.1 \mathrm{~nm}$, respectively. Heterogeneous perturbation of the $D(2)$ and $B(14)$ levels is expected, but occurs at high $J$ values beyond the limit of our observations. The results are presented in Tables Id and Ib for the $\epsilon(2,0)$ and $\beta(14,0)$ bands, respectively. The $\delta(3,0)$ band is observed in the wavelength region of 168.8-169.6 $\mathrm{nm}$ and is presented in Table Ic. The upper level of the $\delta(3,0)$ band is perturbed strongly by the $B(15)$ level (not observed in these measurements). ${ }^{23}$ The $\epsilon(3,0)$ band is observed in the wavelength region of $166.3-167.2 \mathrm{~nm}$ and is presented in Table Id. We were able to extend the assignment of lines by comparison with Miescher, ${ }^{24}$ and the band head areas of the $P_{11}$ and $P_{21}+Q_{11}$ branches are reassigned.

The $\mathrm{NO}^{2} \Pi$ multistate interaction is a well-known perturbation that has been examined in detail. ${ }^{11,23}$ The $B^{2} \Pi$ $-C^{2} \Pi$ interaction is so strong that it is insufficient to consider only pairwise $B(12){ }^{2} \Pi-C(2){ }^{2} \Pi$ or $B(15)^{2} \Pi-C(3){ }^{2} \Pi$ vibrational level interaction. Every rotational level of the $B$ $-C$ complex is significantly mixed. A quantitative analysis of the observations in terms of the five electronic states $B^{2} \Pi$, $C^{2} \Pi, K^{2} \Pi, L^{2} \Pi$, and $Q^{2} \Pi$ was performed by Gallusser and Dressler. ${ }^{11}$ The coupling is treated with a vibronic interaction matrix that includes the bound vibrational levels of these five states. Both components of the spin doublets and three different isotopes are included, and the calculated energies and $B$ values of the perturbed vibronic levels are fitted to the experimental data in the determination of RKR potential curves and off-diagonal electronic energy. The results include predicted oscillator strengths for all the bands as well as detailed rotational structures for complex multistate interactions. In view of the comprehensive work performed by Gallusser and Dressler, ${ }^{11}$ we could not undertake any leastsquares fitting with the few bands that we observed in this energy region.
The rotational term values of the upper states were obtained by adding the term values of the $v=0$ level of the $X^{2} \Pi$ state to the wave numbers of the observed transition lines. Accurate rotational term values of the $X^{2} \Pi$ state reported by Amiot et al. ${ }^{25}$ by using high-resolution FT infrared spectroscopy were used, for which the $\Omega=1 / 2, J=0.5$, and $e$ level was taken as the relative zero. The upper-state term values obtained from various branches were averaged only from isolated lines when possible, but the term values are followed by " $\mathrm{B}$ " if all branch lines are blended. The results are listed in Table IIa for the $B(10)^{2} \Pi$ and $C(1)^{2} \Pi$ levels; in Table IIb for the $B^{2} \Pi$ levels with $v=12,14$; in Table IIc for the $C^{2} \Pi$ levels with $v=2,3$; and in Table IId for the $D^{2} \Sigma$ levels with $v=2,3$.

Table IIa for the $B(10,0)$ levels also includes the $C(1)$ levels, most of which were published in an earlier paper, ${ }^{2}$ because the relevant spectrum was taken at a much higher column density, allowing us to extend the measurements to weak high $J$ lines of the $\delta(1,0)$ band. The term values for $B(10)$ and $C(1)$ are plotted against $J(J+1)$ in Fig. 1 . The two curves cross around $J=19.5$, corresponding to $J(J+1)=400$, and the mixing of intensity is discussed in Sec. III B. The $B(12)$ levels (Table IIb) are perturbed strongly by the $C(2)$ level, and so it is not possible to present the molecular constants. Gallusser and Dressler ${ }^{11}$ calculated the perturbed rotational constants at $J=0$. Their values are 1.524 and $1.484 \mathrm{~cm}^{-1}$ for the $F_{1}$ and $F_{2}$ levels of the $C(2)$ level, and our observed values are 1.439 and $1.583 \mathrm{~cm}^{-1}$. Their values for the $B(12)$ level are 1.267 and $1.311 \mathrm{~cm}^{-1}$ for the $F_{1}$ and $F_{2}$ levels, and our observed values are 1.230 and $1.377 \mathrm{~cm}^{-1}$. The molecular constants of the $B(14)$ levels are presented in Table IIb, because the heterogeneous interaction with the $D(2)$ level occurs at high $J$ beyond of our observation. The $C$ (3) levels are also strongly perturbed, and the molecular constants are not presented. The heterogeneous perturbation of the ${ }^{2} \Sigma-{ }^{2} \Pi$ states of NO was discussed by Jungen and Miescher. ${ }^{26}$ Fitting for the molecular constants is limited to terms up to $J=15.5$ and 20.5 for the $D(2)$ and $D(3)$ levels, respectively, because the high $J$ levels are shifted by the heterogeneous perturbation of the $B(14)$ and $B(16)$ levels.

The term values reported by Braun et al. ${ }^{13}$ for the $v=2$ levels can be compared with our $C(2)$ and $D(2)$ levels. The absolute values of Braun et al. depend on the UV measurements of the older grating measurements of Engleman and Rouse $^{27}$ with an uncertainty of $0.1 \mathrm{~cm}^{-1}$. Our values for the $v=2$ levels are larger than those of Braun et al. by $0.08 \pm 0.03 \mathrm{~cm}^{-1}$ on average, well within the combined uncertainties. We reported in Ref. 7 that the mean difference between our values for the $v=0,1$ levels and those of Braun et al. is only $0.017 \mathrm{~cm}^{-1}$.

\section{B. Integrated cross sections and oscillator strengths}

The fitting procedure employed under GREMLIN Ref. 19 also evaluates areas and hence integrated cross sections for the lines fitted. However, these are distorted by the instrumental function, as stated in Sec. II. This effect was not taken into account for our previously published integrated cross sections, ${ }^{1-6}$ the band oscillator strengths of which are 
(a)

$\beta(10,0)$ band $[B(10)-X(0)]$

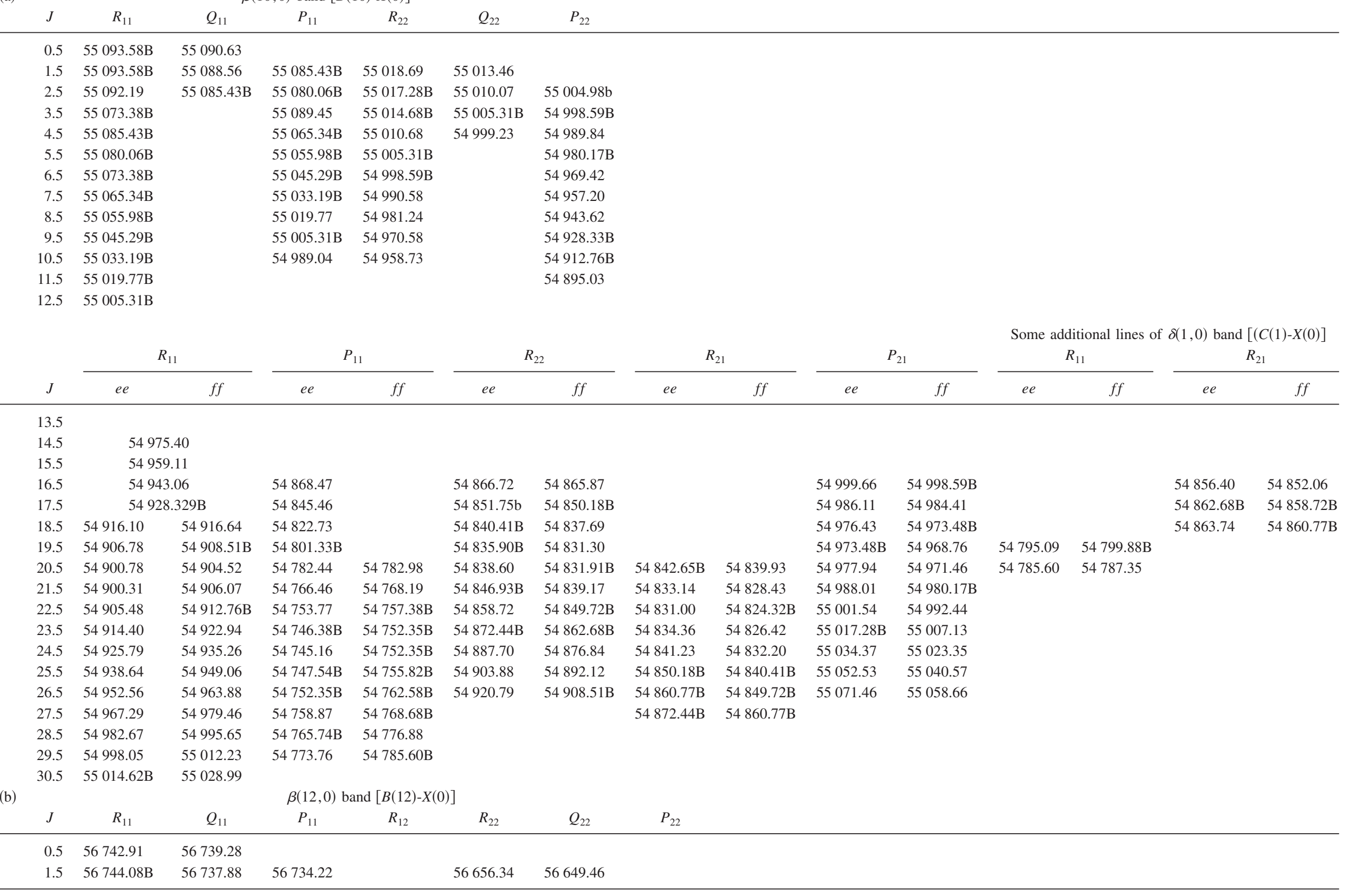




\begin{tabular}{|c|c|c|c|c|c|c|c|}
\hline 2.5 & $56744.08 \mathrm{~B}$ & 56735.64 & 56729.54 & $56623.99 \mathrm{~B}$ & $56657.45 \mathrm{~B}$ & 56647.75 & 56640.84 \\
\hline 3.5 & 56743.37 & 56732.49 & $56723.82 \mathrm{~B}$ & $56623.14 \mathrm{~B}$ & $56657.45 \mathrm{~B}$ & 56645.32 & 56635.70 \\
\hline 4.5 & 56741.60 & 56728.37 & 56717.42 & 56620.11 & $56656.86 \mathrm{~B}$ & $56641.81 \mathrm{~B}$ & 56629.85 \\
\hline 6.5 & 56734.95 & & 56701.48 & 56612.94 & 56652.46 & 56632.81 & 56615.57 \\
\hline 7.5 & 56729.96 & & 56691.99 & $56607.05 \mathrm{~B}$ & 56648.60 & 56626.66 & $56607.05 \mathrm{~B}$ \\
\hline 8.5 & $56723.82 \mathrm{~B}$ & & 56681.45 & 56600.21 & 56643.50 & & 56597.46 \\
\hline 9.5 & 56716.30 & & 56669.76 & & 56637.07 & & 56586.73 \\
\hline 10.5 & 56707.51 & & $56656.86 \mathrm{~B}$ & & 56629.24 & & 56574.78 \\
\hline 11.5 & 56697.35 & & 56642.74 & & 56619.88 & & 56561.50 \\
\hline 12.5 & 56685.73 & & 56627.27 & & 56609.06 & & 56546.80 \\
\hline 13.5 & 56672.62 & & 56610.42 & & 56596.56 & & 56530.65 \\
\hline 14.5 & 56657.99 & & 56592.11 & & 56582.42 & & 56512.94 \\
\hline 15.5 & $56641.81 \mathrm{~B}$ & & 56572.32 & & 56566.64 & & 56493.62 \\
\hline 16.5 & $56623.99 \mathrm{~B}$ & & 56551.01 & & 56549.17 & & 56472.66 \\
\hline 17.5 & 56604.57 & & 56528.11 & & 56529.93 & & 56450.00 \\
\hline 18.5 & 56583.53 & & 56503.66 & & 56508.88 & & \\
\hline \multirow[t]{2}{*}{20.5} & 56536.67 & & & & & & \\
\hline & \multicolumn{7}{|c|}{$\beta(14,0)$ band $[B(14)-X(0)]$} \\
\hline$J$ & $R_{11}$ & $Q_{11}$ & $P_{11}$ & $R_{22}$ & $Q_{22}$ & $P_{22}$ & $R_{21}$ \\
\hline 0.5 & $58542.03 \mathrm{~B}$ & 58538.97 & & & & & \\
\hline 1.5 & $58542.03 B$ & 58536.99 & $58533.96 \mathrm{~B}$ & 58471.05 & 58465.72 & & \\
\hline 2.5 & 58540.78 & $58533.96 \mathrm{~B}$ & 58528.64 & 58469.94 & 58462.47 & 58457.12 & $58589.76 \mathrm{w}$ \\
\hline 3.5 & 58538.20 & $58529.06 \mathrm{~B}$ & 58521.99 & 58467.49 & 58457.89 & 58450.42 & $58588.71 w$ \\
\hline 4.5 & 58534.31 & & 58514.05 & 58463.70 & $58452.11 \mathrm{~B}$ & 58442.42 & $58584.73 w$ \\
\hline 5.5 & 58529.06 & 58515.95 & 58504.77 & 58458.60 & 58444.82 & 58433.09 & $58579.76 w$ \\
\hline 6.5 & 58522.48 & & 58494.18 & 58452.11 & 58436.25 & 58422.45 & $58573.55 w$ \\
\hline 7.5 & 58514.55 & & 58482.25 & 58444.31 & 58426.29 & 58410.45 & $58566.64 w$ \\
\hline 8.5 & 58505.27 & & 58469.02 & 58435.11 & 58415.06 & 58397.12 & $58558.52 w$ \\
\hline 9.5 & 58494.65 & & 58454.33 & 58424.53 & & $58382.40 \mathrm{~B}$ & $58549.12 w$ \\
\hline 10.5 & 58482.61 & & $58438.40 \mathrm{~B}$ & 58412.57 & & 58366.39 & \\
\hline 11.5 & 58469.35 & & $58421.05 B$ & 58399.20 & & 58348.95 & \\
\hline 12.5 & 58454.49 & & 58 402.36B & 58384.42 & & 58330.13 & \\
\hline 16.5 & $58382.40 \mathrm{~B}$ & & 58313.91 & 58311.05 & & $58240.86 \mathrm{~B}$ & \\
\hline 17.5 & 58 360.94B & & 58 288.30B & 58289.15 & & 58214.90 & \\
\hline 18.5 & $58338.13 \mathrm{~B}$ & & 58261.43 & 58 265.27B & & 58187.57 & \\
\hline 19.5 & & & 58232.89 & 58 240.86B & & 58158.92 & \\
\hline 20.5 & & & 58203.59 & & & 58128.81 & \\
\hline
\end{tabular}




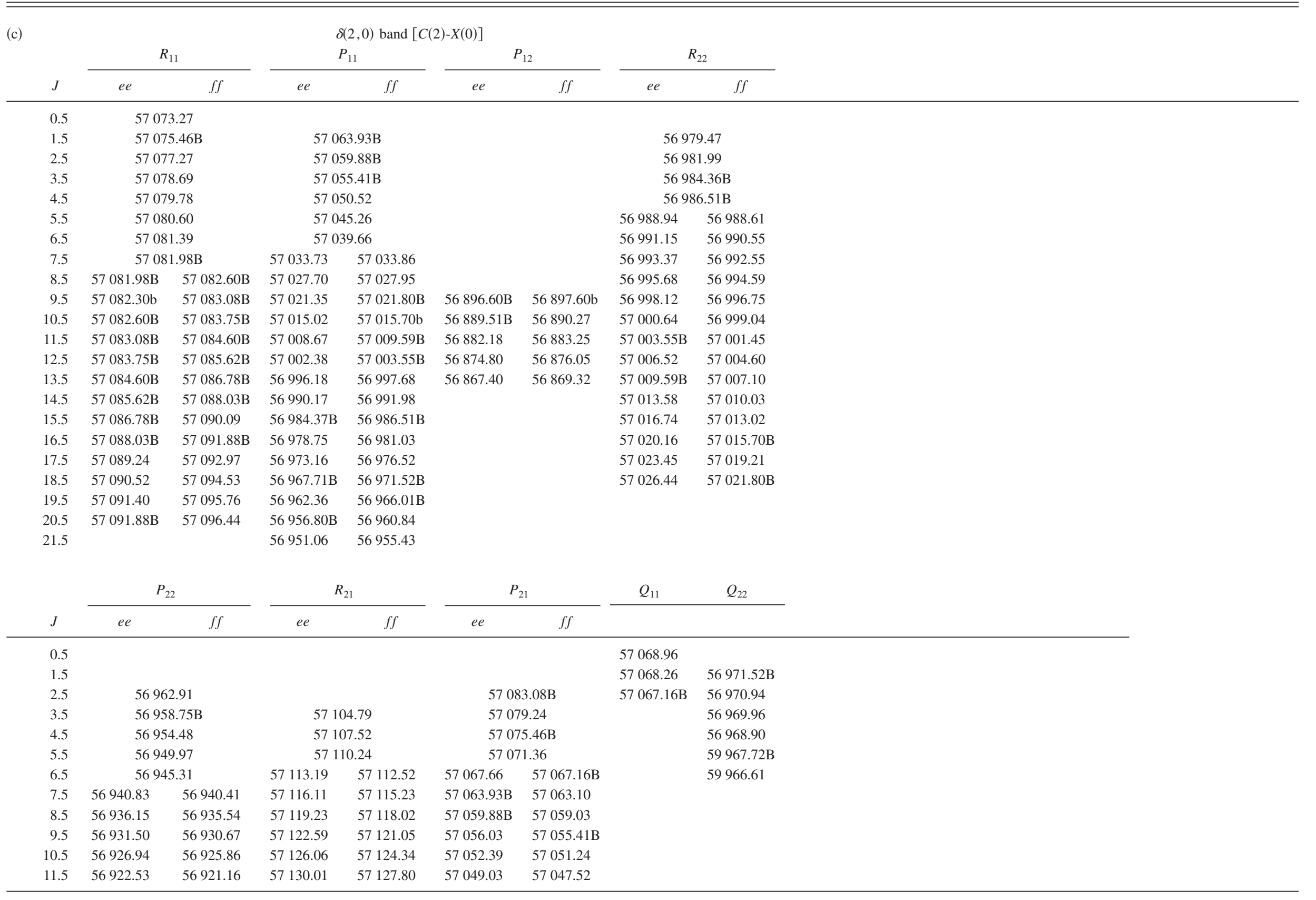




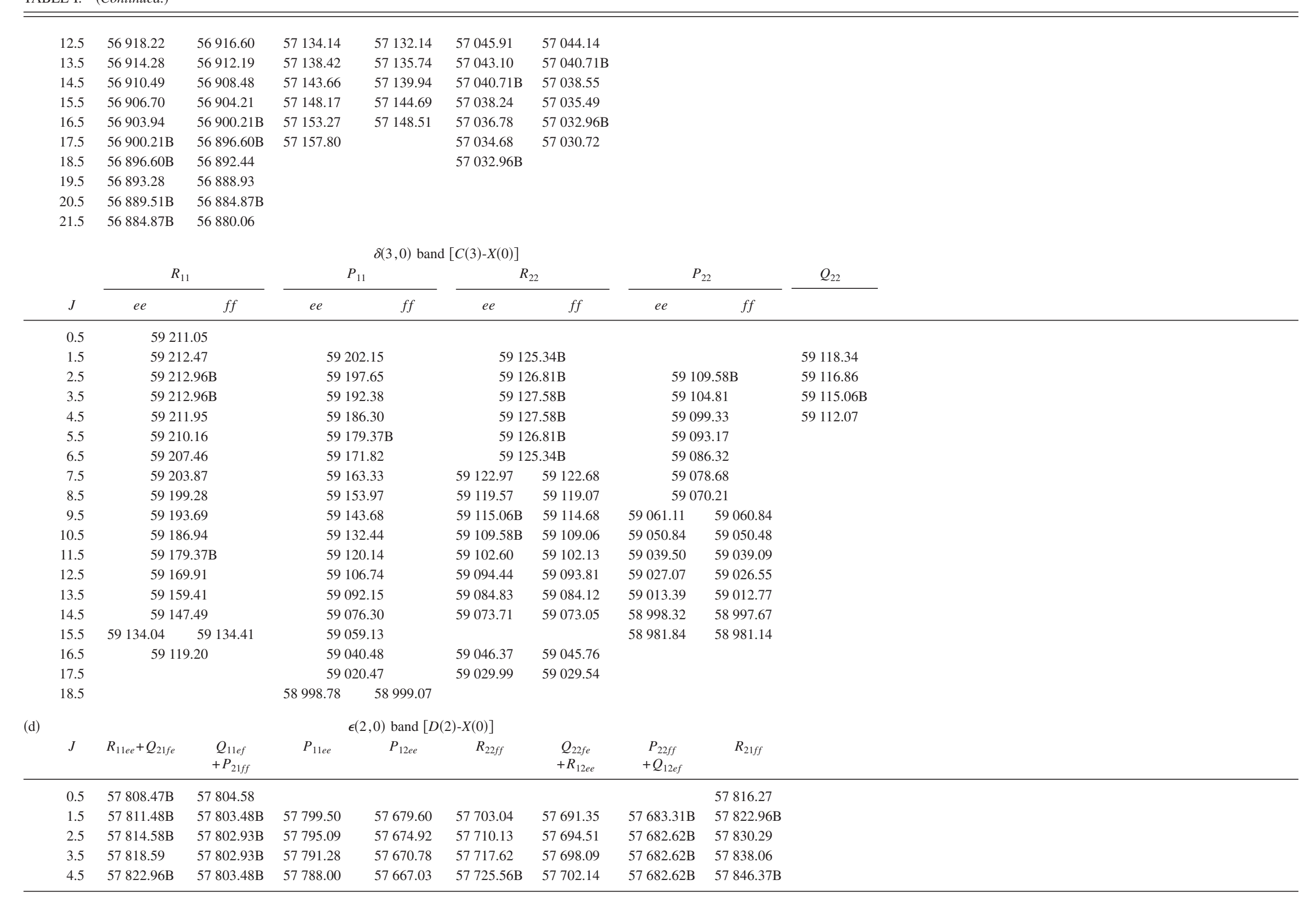

$\delta(3,0)$ band $[C(3)-X(0)]$ 


\begin{tabular}{|c|c|c|c|c|c|c|c|c|}
\hline 5.5 & 57828.09 & $57804.58 \mathrm{~B}$ & 57785.25 & 57663.75 & 57733.92 & 57706.64 & $57683.31 \mathrm{~B}$ & 57 855.32B \\
\hline 6.5 & 57833.65 & 57806.30 & 57783.01 & 57660.93 & 57742.81 & 57711.56 & 57684.30 & 57864.77 \\
\hline 7.5 & 57839.73 & $57808.47 \mathrm{~B}$ & 57781.35 & 57658.52 & 57752.02 & 57716.95 & 57685.81 & 57874.68 \\
\hline 8.5 & $57846.37 \mathrm{~B}$ & $57811.48 \mathrm{~B}$ & 57 780.06B & 57656.58 & 57761.69 & 57722.79 & 57687.72 & 57885.21 \\
\hline 9.5 & 57853.53 & $57814.58 \mathrm{~B}$ & 57 779.54B & 57655.08 & 57771.88 & 57729.07 & 57690.12 & 57896.24 \\
\hline 10.5 & 57861.24 & 57818.35 & 57 779.54B & 57654.07 & $57782.53 \mathrm{~B}$ & 57735.79 & 57692.98 & 57 907.76B \\
\hline 11.5 & 57869.48 & 57822.70 & 57 780.06B & $57653.43 \mathrm{~B}$ & 57793.52 & 57742.89 & 57696.28 & 57919.90 \\
\hline 12.5 & 57878.23 & 57827.55 & 57781.04 & $57653.43 \mathrm{~B}$ & 57805.07 & 57750.60 & 57700.03 & 57932.53 \\
\hline 13.5 & 57887.53 & 57832.93 & 57 782.53B & $57653.43 \mathrm{~B}$ & 57816.99 & 57758.67 & 57704.25 & 57945.69 \\
\hline 14.5 & 57897.38 & 57838.89 & 57784.64 & 57654.44 & 57829.39 & 57767.24 & 57708.88 & 57959.40 \\
\hline 15.5 & 57 907.76B & 57845.37 & 57787.26 & 57655.70 & 57842.28 & 57776.21 & 57714.01 & 57973.71 \\
\hline 16.5 & 57918.56 & 57852.36 & 57790.35 & & 57 855.32B & 57785.67 & 57719.58 & 57988.31 \\
\hline 17.5 & 57929.94 & 57859.89 & 57794.07 & & & 57795.55 & $57725.56 \mathrm{~B}$ & 58003.55 \\
\hline 18.5 & 57941.84 & 57867.91 & 57798.23 & & & 57805.85 & 57732.11 & 58019.29 \\
\hline 19.5 & 57954.24 & 57876.48 & & & & 57816.64 & 57738.99 & 58035.45 \\
\hline 20.5 & 57967.10 & 57885.53 & & & & & 57746.38 & \\
\hline 21.5 & 57980.52 & 57895.06 & & & & & 57754.16 & \\
\hline 22.5 & 57994.24 & 57905.04 & & & & & & \\
\hline \multirow[t]{2}{*}{23.5} & 58008.39 & & & & & & & \\
\hline & \multicolumn{8}{|c|}{$\epsilon(3,0)$ band $[D(3)-X(0)]$} \\
\hline$J$ & $\begin{aligned} & R_{11 e e} \\
+ & Q_{21 f e}\end{aligned}$ & $\begin{array}{c}Q_{11 e f} \\
+P_{21 f f}\end{array}$ & $P_{11 e e}$ & $P_{12 e e}$ & $R_{22 f f}$ & $\begin{array}{c}Q_{22 f e} \\
+R_{12 e e}\end{array}$ & $\begin{array}{c}P_{22 f f} \\
+Q_{12 e f}\end{array}$ & $R_{21 f f}$ \\
\hline 0.5 & 59991.11 & 59987.26 & & & & & & 59998.82 \\
\hline 1.5 & 59993.81 & 59986.10 & 59982.26 & 59862.31 & $59885.27 \mathrm{~B}$ & 59873.88 & 59866.21 & 60005.36 \\
\hline 2.5 & 59996.99 & 59 985.34B & 59977.74 & 59857.60 & 59892.20 & 59876.83 & 59865.35 & 60012.38 \\
\hline 3.5 & 60000.69 & $59985.34 \mathrm{~B}$ & 59973.77 & 59853.24 & 59899.39 & 59880.20 & $59864.87 \mathrm{~B}$ & 60019.90 \\
\hline 4.5 & 60004.90 & 59985.52 & 59970.25 & 59849.30 & 59907.04 & 59883.93 & $59864.87 \mathrm{~B}$ & 60027.94 \\
\hline 5.5 & 60009.59 & 59986.44 & $59967.28 \mathrm{~B}$ & 59845.80 & $59914.86 \mathrm{~B}$ & 59888.13 & 59865.10 & 60036.44 \\
\hline 6.5 & 60014.78 & 59987.76 & 59964.78 & 59842.70 & 59923.43 & $59892.68 \mathrm{~B}$ & 59865.79 & 60045.43 \\
\hline 7.5 & $60020.41 \mathrm{~B}$ & 59989.62 & 59962.77 & 59839.99 & 59932.22 & 59897.65 & 59866.90 & 60054.92 \\
\hline 8.5 & 60026.59 & $59991.93 \mathrm{~B}$ & 59961.29 & 59837.66 & 59941.38 & 59903.00 & 59868.44 & 60064.87 \\
\hline 9.5 & 60033.19 & 59994.71 & 59960.34 & 59835.78 & 59950.89 & 59908.72 & 59870.34 & 60075.27 \\
\hline 10.5 & 60040.28 & 59997.98 & $59959.70 \mathrm{~B}$ & 59834.29 & $59960.83 B$ & $59914.86 \mathrm{~B}$ & 59872.65 & 60086.08 \\
\hline 11.5 & 60047.77 & 60001.70 & $59959.70 \mathrm{~B}$ & 59833.12 & 59970.88 & 59921.26 & 59875.31 & 60097.33 \\
\hline 12.5 & 60055.67 & 60005.85 & 59960.13 & $59832.46 \mathrm{~B}$ & 59981.36 & 59928.03 & 59878.30 & 60108.88 \\
\hline 13.5 & 60063.91 & 60010.38 & 59 960.83B & $59831.97 \mathrm{~B}$ & 59 991.93B & 59935.05 & 59881.67 & 60120.66 \\
\hline 14.5 & 60072.38 & 60015.29 & 59962.07 & $59831.97 \mathrm{~B}$ & & 59942.19 & $59885.27 \mathrm{~B}$ & 60132.39 \\
\hline 15.5 & 60080.84 & $60020.41 \mathrm{~B}$ & 59963.67 & $59831.97 \mathrm{~B}$ & & 59949.25 & 59889.03 & \\
\hline 16.5 & 60088.97 & 60025.59 & 59965.47 & $59832.46 \mathrm{~B}$ & & 59956.03 & $59892.68 \mathrm{~B}$ & \\
\hline 17.5 & & 60030.31 & $59967.28 \mathrm{~B}$ & & & & & \\
\hline 18.5 & & 60034.11 & & & & & & \\
\hline
\end{tabular}


TABLE II. Observed term values of the $B(10,12,14), C(2,3)$, and $D(2,3)$ levels of NO. Values followed by B are obtained from blended lines. Molecular constants are given for the $B(14), D(2)$, and $D(3)$ levels. (a) The term values of the $B(10)$ and $C(1)$ levels of $N O$ (in $\mathrm{cm}^{-1}$ ). (b) The term values of the $B(12$ ) and $B(14)$ levels of $\mathrm{NO}\left(\mathrm{in} \mathrm{cm}^{-1}\right.$ ). (c) The term values of the $C(2)$ and $C(3)$ levels of $\mathrm{NO}$ (in $\mathrm{cm}^{-1}$ ). (d) The term values of $D(2)$ and $D(3)$ levels of $\mathrm{NO}$ (in $\left.\mathrm{cm}^{-1}\right)$.

(a)

\begin{tabular}{|c|c|c|c|c|c|c|c|c|}
\hline \multirow[b]{2}{*}{$J$} & \multicolumn{2}{|c|}{$C(1) F_{1} \rightarrow B(10) F_{1}$} & \multicolumn{2}{|c|}{$C(1) F_{2} \rightarrow B(10) F_{2}$} & \multicolumn{2}{|c|}{$B(10) F_{1} \rightarrow C(1) F_{1}$} & \multicolumn{2}{|c|}{$B(10) F_{2} \rightarrow C(1) F_{2}$} \\
\hline & $e$ & $f$ & $e$ & $f$ & $e$ & $f$ & $e$ & $f$ \\
\hline 0.5 & 54690.00 & 54690.01 & & & 55090.44 & 55090.46 & & \\
\hline 1.5 & 54692.61 & 54692.63 & 54699.33 & 54699.35 & 55093.50 & 55093.53 & 55138.50 & 55138.50 \\
\hline 2.5 & 54700.18 & 54700.19 & 54711.12 & 54711.12 & 55098.52 & 55098.55 & 55143.61 & 55143.61 \\
\hline 3.5 & 54711.66 & 54711.70 & 54726.85 & 54726.54 & 55105.50 & 55105.55 & 55150.84 & 55150.84 \\
\hline 4.5 & 54727.07 & 54727.08 & 54746.28 & 54745.82 & 55114.49 & 55114.55 & 55160.11 & 55160.11 \\
\hline 5.5 & 54746.19 & 54746.66 & 54769.60 & 54768.94 & 55125.52 & 55125.59 & 55171.71 & 55171.71 \\
\hline 6.5 & 54769.24 & 54769.88 & 54796.77 & 54795.88 & 55138.52 & 55138.60 & 55185.27 & 55185.27 \\
\hline 7.5 & 54796.18 & 54797.02 & 54827.78 & 54826.64 & 55153.54 & 55153.63 & 55200.90 & 55200.91 \\
\hline 8.5 & 54826.92 & 54828.00 & 54862.63 & 54861.22 & 55170.71 & 55170.81 & 55218.67 & 55218.67 \\
\hline 9.5 & 54861.45 & 54862.82 & 54901.28 & 54899.58 & 55189.65 & 55189.76 & 55238.66 & 55238.67 \\
\hline 10.5 & 54899.81 & 54901.47 & 54943.74 & 54941.71 & 55210.76 & 55210.88 & 55260.54 & 55260.55 \\
\hline 11.5 & 54941.93 & 54943.91 & 54989.96 & 54987.56 & 55233.77 & 55233.89 & 55284.76 & 55284.76 \\
\hline 12.5 & 54987.76 & 54990.09 & 55039.84 & 55037.08 & & & & \\
\hline 13.5 & 55037.26 & 55039.98 & 55093.32 & 55090.18 & & & & \\
\hline 14.5 & 55090.36 & 55093.44 & 55150.25 & 55146.73 & & & & \\
\hline 15.5 & 55146.90 & 55150.38 & 55210.31 & 55206.48 & 55349.83 & 55350.00 & & \\
\hline 16.5 & 55206.65 & 55210.46 & 55273.20 & 55269.04 & 55385.33 & 55385.51 & 55441.86 & 55440.81 \\
\hline 17.5 & 55269.16 & 55273.25 & 55337.70 & 55333.63 & 55424.43 & 55424.62 & 55481.04 & 55480.18 \\
\hline 18.5 & 55333.72 & 55337.88 & 55402.67 & 55398.79 & 55468.19 & 55468.39 & 55526.02 & 55524.50 \\
\hline 19.5 & 55398.78 & 55403.22 & 55465.43 & 55462.66 & 55517.79 & 55518.54 & 55578.13 & 55575.47 \\
\hline 20.5 & 55461.95 & & 55525.58 & 55523.47 & 55573.65 & 55575.60 & 55640.36 & 55635.84 \\
\hline 21.5 & 55520.96 & 55522.93 & & & 55636.21 & 55640.03 & 55713.33 & 55706.87 \\
\hline 22.5 & 55575.88 & 55577.49 & & & 55707.39 & 55713.46 & 55795.22 & 55787.53 \\
\hline 23.5 & 55628.74 & 55630.13 & & & 55787.85 & 55795.34 & 55883.94 & 55875.07 \\
\hline 24.5 & & & & & 55875.29 & 55883.97 & 55978.09 & 55968.37 \\
\hline 25.5 & & & & & 55968.57 & 55978.19 & 56077.07 & 56066.28 \\
\hline 26.5 & & & & & 56066.74 & 56077.10 & 56180.38 & 56168.67 \\
\hline 27.5 & & & & & 56168.94 & 56180.45 & 56287.78 & 56275.23 \\
\hline 28.5 & & & & & 56275.43 & 56287.84 & & \\
\hline 29.5 & & & & & 56385.91 & 56399.15 & & \\
\hline 30.5 & & & & & & 56514.17 & & \\
\hline
\end{tabular}

(b)

\begin{tabular}{|c|c|c|c|c|c|c|c|c|}
\hline \multirow[b]{2}{*}{$J$} & & \\
\hline & $F_{1 e}$ & $F_{1 f}$ & $F_{2 e}$ & $F_{2 f}$ & $F_{1 e}$ & $F_{1 f}$ & $F_{2 e}$ & $F_{2 f}$ \\
\hline 0.5 & 56739.23 & 56739.24 & & & 58538.97 & 58538.97 & & \\
\hline 1.5 & 56742.91 & 56742.93 & 56774.37 & 56774.37 & 58542.00 & 58542.03 & 58590.62 & 58590.62 \\
\hline 2.5 & 56749.00 & 56749.04 & 56781.26 & 56781.26 & 58547.05 & 58547.10 & 58595.97 & 58595.97 \\
\hline 3.5 & 56757.54 & 56757.58 & 56790.88 & 56790.88 & 58554.15 & 58554.19 & 58603.45 & 58603.45 \\
\hline 4.5 & 56768.44 & 56768.50 & $56802.98 \mathrm{~B}$ & $56802.98 \mathrm{~B}$ & 58563.26 & 58563.32 & 58613.04 & 58613.04 \\
\hline 5.5 & 56781.70 & 56781.77 & 56817.87 & 56817.88 & 58574.40 & 58574.48 & 58624.74 & 58624.74 \\
\hline 6.5 & 56797.30 & 56797.38 & 56835.12 & 56835.12 & 58587.54 & 58587.63 & 58638.54 & 58638.54 \\
\hline 7.5 & 56815.17 & 56815.26 & 56854.76 & 56854.76 & 58602.72 & 58602.81 & 58654.40 & 58654.41 \\
\hline 8.5 & 56835.24 & 56835.34 & 56876.68 & 56876.68 & 58619.81 & 58619.92 & 58672.38 & 58672.38 \\
\hline 9.5 & $56857.52 \mathrm{~B}$ & $56857.63 \mathrm{~B}$ & 56900.81 & 56900.81 & 58638.97 & 58639.08 & 58692.41 & 58692.42 \\
\hline 10.5 & 56881.76 & 56881.89 & 56927.02 & 56927.03 & 58660.05 & 58660.13 & 58714.48 & 58714.49 \\
\hline 11.5 & 56908.08 & 56908.22 & 56955.26 & 56955.26 & 58683.17 & 58683.30 & 58738.59 & 58738.60 \\
\hline 12.5 & 56936.36 & 56936.50 & 56985.43 & 56985.44 & 58708.37 & 58708.50 & 58764.73 & 58764.74 \\
\hline 13.5 & 56966.53 & 56966.68 & 57017.51 & 57017.52 & 58735.30 & 58735.44 & 58792.85 & 58792.87 \\
\hline 14.5 & 56998.55 & 56998.71 & 57051.36 & 57051.37 & $58764.35 \mathrm{~B}$ & $58764.51 \mathrm{~B}$ & 58823.02 & 58823.03 \\
\hline 15.5 & 57032.39 & 57032.57 & 57086.98 & 57087.00 & 58795.27 & 58795.45 & 58855.13 & 58855.15 \\
\hline 16.5 & 57067.98 & 57068.16 & 57124.36 & 57124.38 & $58828.17 \mathrm{~B}$ & $58828.35 \mathrm{~B}$ & 58889.26 & 58889.28 \\
\hline 17.5 & 57105.36 & 57105.55 & 57163.49 & 57163.52 & 58863.11 & 58863.31 & 58925.34 & 58925.35 \\
\hline 18.5 & 57144.44 & 57144.64 & 57204.25 & 57204.28 & 58899.75 & 58899.95 & 58963.45 & 58963.48 \\
\hline
\end{tabular}


TABLE II. (Continued.)

\begin{tabular}{|c|c|c|c|c|c|c|c|c|}
\hline 19.5 & 57185.23 & 57185.43 & 57246.60 & 57246.64 & $58938.95 B$ & 58 939.16B & 59003.52 & 59003.55 \\
\hline 20.5 & 57227.77 & 57227.98 & 57290.85 & 57290.89 & & & $59045.37 \mathrm{~B}$ & 59045.40 \\
\hline \multirow[t]{8}{*}{21.5} & 57272.03 & 57272.25 & & & & & & \\
\hline & & & & & $T_{0}$ & $58563.29(1)$ & & \\
\hline & & & & & $A$ & $50.65(2)$ & & \\
\hline & & & & & $B$ & $1.0381(2)$ & & \\
\hline & & & & & $D \times 10^{4}$ & $4.11(5)$ & & \\
\hline & & & & & $A_{D}$ & $0.0060(2)$ & & \\
\hline & & & & & $p$ & $0.012(2)$ & & \\
\hline & & & & & $q \times 10^{4}$ & 0.411 fixed & & \\
\hline
\end{tabular}

(c)

\begin{tabular}{|c|c|c|c|c|c|c|c|c|}
\hline \multirow[b]{2}{*}{$J$} & \multicolumn{4}{|c|}{$C(2)$} & \multicolumn{4}{|c|}{$C(3)$} \\
\hline & $F_{1 e}$ & $F_{1 f}$ & $F_{2 e}$ & $F_{2 f}$ & $F_{1 e}$ & $F_{1 f}$ & $F_{2 e}$ & $F_{2 f}$ \\
\hline 0.5 & 57 068.96B & 57 068.96B & & & 59207.16 & 59207.19 & & \\
\hline 1.5 & $57073.28 b$ & 57 073.28B & 57 096.43B & 57 096.44B & 59211.03 & 59211.06 & 59243.26 & 59243.26 \\
\hline 2.5 & $57080.47 \mathrm{~B}$ & $57080.51 \mathrm{~B}$ & $57104.36 \mathrm{~B}$ & 57 104.37B & 59217.46 & 59217.50 & 59250.37 & 59250.37 \\
\hline 3.5 & 57 090.63B & 57 090.68B & 57 115.52B & 57 115.54B & 59226.41 & 59226.46 & 59260.36 & 59260.36 \\
\hline 4.5 & 57 103.75B & 57 103.81B & 57 129.94B & 57 129.95B & $59237.94 \mathrm{~B}$ & $59238.00 \mathrm{~B}$ & 59273.11 & 59273.11 \\
\hline 5.5 & $57119.88 \mathrm{~B}$ & 57 119.95B & 57 147.66B & 57 147.59B & 59252.05 & 59252.12 & 59288.61 & 59288.62 \\
\hline 6.5 & 57139.03 & 57139.24 & 57168.90 & 57168.51 & 59268.64 & 59268.72 & 59306.77 & 59306.77 \\
\hline 7.5 & 57161.41 & 57161.76 & 57193.43 & 57192.84 & 59287.68 & 59287.77 & 59327.51 & 59327.51 \\
\hline 8.5 & 57186.82 & 57 187.37B & 57221.45 & 57220.62 & 59309.16 & 59309.26 & 59351.06 & 59350.78 \\
\hline 9.5 & 57215.60 & 57216.30 & 57252.96 & 57251.89 & 59333.00 & 59333.12 & 59376.87 & 59376.45 \\
\hline 10.5 & 57247.70 & 57248.78 & 57288.06 & 57286.68 & 59359.16 & 59359.28 & 59405.03 & 59404.63 \\
\hline 11.5 & 57283.22 & 57284.51 & 57326.70 & 57325.07 & 59387.53 & 59387.66 & 59435.52 & 59435.05 \\
\hline 12.5 & 57322.16 & 57323.77 & 57369.05 & 57366.98 & 59418.09 & 59418.24 & 59468.16 & 59467.63 \\
\hline 13.5 & 57364.58 & 57366.55 & 57414.99 & 57413.08 & 59450.72 & 59450.87 & 59502.89 & 59502.26 \\
\hline 14.5 & 57 410.56B & 57 412.89B & 57464.42 & 57461.90 & 59485.35 & 59485.21 & 59539.61 & 59538.91 \\
\hline 15.5 & 57460.13 & 57462.59 & 57518.16 & 57514.56 & 59521.88 & 59522.05 & 59578.27 & 59577.62 \\
\hline 16.5 & 57513.03 & 57516.53 & 57574.47 & 57570.78 & 59560.29 & 59560.66 & & \\
\hline 17.5 & $57569.40 \mathrm{~B}$ & $57573.42 \mathrm{~B}$ & 57634.57 & 57631.13 & 59600.52 & 59600.86 & 59660.69 & 59660.11 \\
\hline 18.5 & 57629.17 & 57633.02 & 57697.74 & 57693.52 & & & 59704.31 & 59703.90 \\
\hline 19.5 & 57692.21 & 57696.42 & 57764.16 & $57759.61 \mathrm{~B}$ & & & & \\
\hline 20.5 & 57758.26 & 57762.83 & $57833.18 \mathrm{~B}$ & 57828.41 & & & & \\
\hline 21.5 & $57827.24 \mathrm{~B}$ & 57832.01 & & & & & & \\
\hline
\end{tabular}

(d)

\begin{tabular}{rlllll} 
& \multicolumn{3}{c}{$D(2)$} & & \multicolumn{2}{c}{$D(3)$} \\
\cline { 2 - 3 } \cline { 5 - 6 }$J$ & \multicolumn{1}{c}{$F_{1 e}$} & \multicolumn{1}{c}{$F_{2 f}$} & & \multicolumn{1}{c}{$F_{2 e}$} & \multicolumn{1}{c}{$F_{2 f}$} \\
\hline 0.5 & 57804.52 & $57808.49 \mathrm{~B}$ & & 59987.26 & $59991.12 \mathrm{~B}$ \\
1.5 & 57808.43 & 57816.28 & & 59991.12 & 59998.82 \\
2.5 & 57816.34 & 57827.95 & & 59998.81 & 60010.37 \\
3.5 & 57828.08 & 57843.67 & & 60010.35 & 60025.73 \\
4.5 & 57843.70 & 57863.18 & & 60025.75 & 60044.95 \\
5.5 & 57863.23 & $57886.58 \mathrm{~B}$ & & 60045.00 & 60068.06 \\
6.5 & 57886.61 & 57913.87 & & 60068.07 & 60094.92 \\
7.5 & 57913.88 & 57945.07 & & 60094.98 & 60125.69 \\
8.5 & 57945.03 & 57980.11 & & 60125.79 & 60160.26 \\
9.5 & 57980.09 & 58018.99 & & 60160.32 & 60198.63 \\
10.5 & $58019.01 \mathrm{~B}$ & 58061.83 & & 60198.65 & 60240.81 \\
11.5 & $58061.84 \mathrm{~B}$ & $58108.50 \mathrm{~B}$ & $60240.88 \mathrm{~B}$ & 60286.65 \\
12.5 & $58108.47 \mathrm{~B}$ & 58159.05 & & $60286.78 \mathrm{~B}$ & 60336.38 \\
13.5 & 58159.03 & 58213.48 & & 60336.48 & 60389.76 \\
14.5 & 58213.46 & 58271.79 & 60389.89 & 60446.60 \\
15.5 & 58271.73 & 58333.97 & 60446.85 & 60506.80 \\
16.5 & $58333.96 \mathrm{~B}$ & $58399.99 \mathrm{~B}$ & $60507.07 \mathrm{~B}$ & $60570.35 \mathrm{~B}$ \\
17.5 & $58399.94 \mathrm{~B}$ & 58469.87 & $60570.36 \mathrm{~B}$ & $60635.80 \mathrm{~B}$ \\
18.5 & $58469.83 \mathrm{~B}$ & 58543.61 & $60636.00 \mathrm{~B}$ & \\
19.5 & $58543.55 \mathrm{~B}$ & 58621.18 & &
\end{tabular}


TABLE II. (Continued.)

\begin{tabular}{ccccc}
\hline \hline & & & \\
20.5 & $58621.12 \mathrm{~B}$ & 58702.52 & & \\
21.5 & $58702.49 \mathrm{~B}$ & $58787.67 \mathrm{~B}$ & & \\
22.5 & $58787.67 \mathrm{~B}$ & $58876.61 \mathrm{~B}$ & & \\
23.5 & $58876.61 \mathrm{~B}$ & $58969.25 \mathrm{~B}$ & & \\
24.5 & $58969.25 \mathrm{~B}$ & & & \\
& $T_{0}$ & $57804.66(2)$ & $T_{0}$ & $59987.21(3)$ \\
& $B$ & $1.9499(2)$ & $B$ & $1.9284(6)$ \\
& $D$ & $1.4(6) \times 10^{-5}$ & $D$ & $5.5(3) \times 10^{-5}$ \\
\hline \hline
\end{tabular}

therefore too low by amounts ranging from $2 \%$ to $20 \%$. The correction described at the end of Sec. II has been applied to the cross sections of all rotational lines of all observed bands, including those previously published. The results are presented in Table III. The integrated cross sections of blended lines, followed by " $b$ " in Table III, have been obtained by using branching ratios observed for other transitions together with the Boltzmann population distribution. The values listed in these tables can be divided by the fractional populations of the rotational levels to obtain values proportional to the line oscillator strengths.

The integrated cross sections of the the $\delta(0,0)$ and $\beta(7,0)$ bands are given in Table IIIa. These values supersede our earlier values, ${ }^{8}$ because of the better experimental conditions and higher signal-to-noise ratios. The upper levels of both bands are homogeneously perturbed at very low $J$, and the branch lines with the $F_{2}^{\prime}$ level of the $\beta(7,0)$ band appear stronger than those with the $F_{1}^{\prime}$ level because of the stronger interaction for the $F_{2}^{\prime}$ level.

The results for the $\delta(1,0)$ and $\beta(10,0)$ bands are presented in Table IIIb. The intensities are affected by the interaction between the upper levels $C(1)$ and $B(10)$, as shown by the plot of the term values in Fig. 1. The two lower curves [the $F_{1}$ and $F_{2}$ components of the $C(1)$ level] are taken from our previous report ${ }^{2}$ and the two upper curves [the $F_{1}$ and $F_{2}$ components of the $B(10)$ level] from the present work. The rotational levels of $B(10)$ with $J \geqslant 13$ belong really to the extension of the $C(1)$ level. Therefore the intensities of lines with $J \geqslant 13$ should be treated as belonging to the $\delta(1,0)$ band.

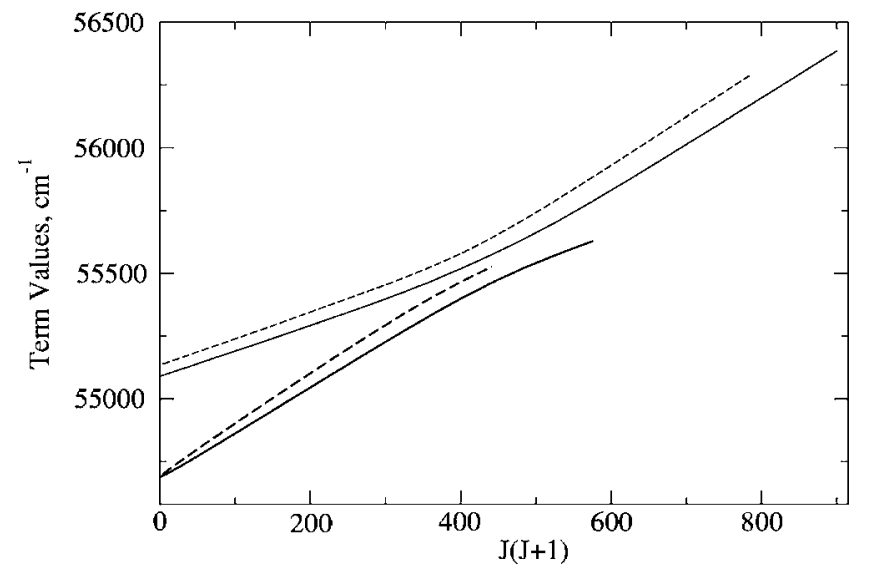

FIG. 1. The term values of the $C(1)$ and $B(10)$ levels plotted against $J(J$ +1 ). The solid curves are the $F_{1}$ component and the broken curves are the $F_{2}$ component. The heavy curves are the $C(1)$ level and the thin curves are the $B(10)$ levels.
The integrated cross sections of the $\beta(6,0), \beta(9,0)$, $\beta(11,0), \beta(12,0)$, and $\beta(14,0)$ bands are presented in Table IIIc. The $B(12)$ level, the upper level of the $\beta(12,0)$ band, is homogeneously perturbed by the $C(2)$ level, the upper level of the $\delta(2,0)$ band. The bands appear separately as two independent bands, but their separation comes from the strong interaction. The $\beta(12,0)$ band appears ten times stronger than the other $\beta$ bands because of the enhancement of its intensity from the $\delta(2,0)$ band.

The integrated cross sections of the $\epsilon(v, 0)$ bands with $v=0-3$ are presented in Table IIId. The results for the $\epsilon(0,0)$ and $\epsilon(1,0)$ bands supersede our earlier values. ${ }^{3,6}$ The upper state of these bands, the $D^{2} \Sigma$ state, could interact heterogeneously with the $B^{2} \Pi$ state, but the interaction occurs at high $J$ levels beyond our observations.

The results for the $\delta(2,0)$ and $\delta(3,0)$ bands are presented in Table IIIe. The upper levels of $C(2)$ and $C(3)$ are homogeneously perturbed by the $B(12)$ and $B(15)$ levels, respectively. The integrated cross sections of the $P_{11}$ branch lines of the $\delta(2,0)$ and $\beta(12,0)$ bands are plotted against $J^{\prime \prime}$ in Fig. 2. The open diamonds and circles represent the integrated cross sections of the $\delta(2,0)$ and $\beta(12,0)$ bands, respectively. The peak intensities appear at $J \sim 10$ and $J \sim 5$ for the $\delta(2,0)$ and $\beta(12,0)$ bands, respectively. These shifts from the Boltzmann population distribution come from the perturbation between the $C(2)$ and $B(12)$ levels. The sum of the branch lines of both bands is plotted as the open squares in the same figure, and this represents the normal Boltzmann population.

The integrated cross sections of the $\gamma(3,0)$ band are presented in Table IIIf. These values supersede our earlier values. $^{4}$

The uncertainties in the integrated cross sections arise from noise and the errors in the measurement of path length and pressure. For the unblended strong lines, the uncertainty due to noise is estimated to be $3 \%-4 \%$, and for the weaker lines it is about $10 \%$. The larger uncertainties of weaker lines do not significantly affect the total integrated cross section of the band, because this is determined predominantly by the strong lines. The uncertainty in the total integrated cross section for the band is estimated to be about $5 \%$.

The band oscillator strength of a $\left(v^{\prime}, v^{\prime \prime}\right)$ band is given by

$$
f\left(v^{\prime}, v^{\prime \prime}\right)=\frac{m c^{2}}{\pi e^{2}} \frac{1}{\tilde{N}\left(v^{\prime \prime}\right)} \int \sigma(\nu) d \nu,
$$

in which $\tilde{N}\left(v^{\prime \prime}\right)$ is the fractional Boltzmann population of the absorbing vibrational level-in this case since $v^{\prime \prime}=0$ this 


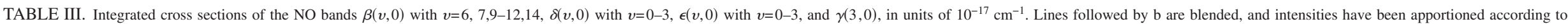

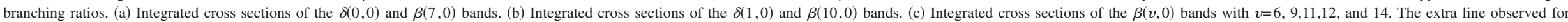
the $\beta(9,0)$ band are listed under extra. (d) Integrated cross sections of the $\epsilon(v, 0)$ bands with $v=0-3$. (e) Integrated cross sections of the $\delta(2,0)$ and $\delta(3,0)$ bands. (f) Integrated cross sections of the $\gamma(3,0)$ band.

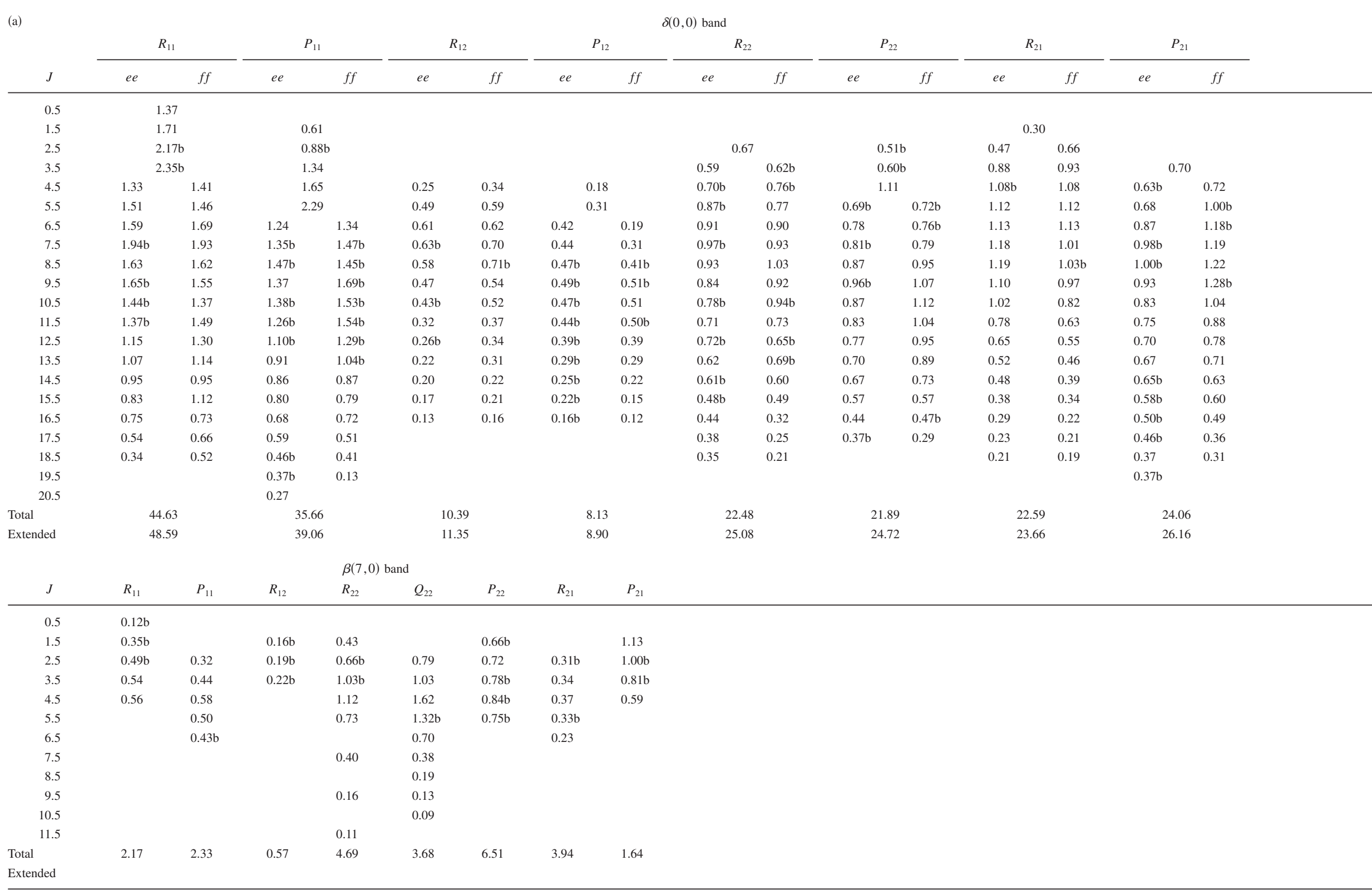

Extended 


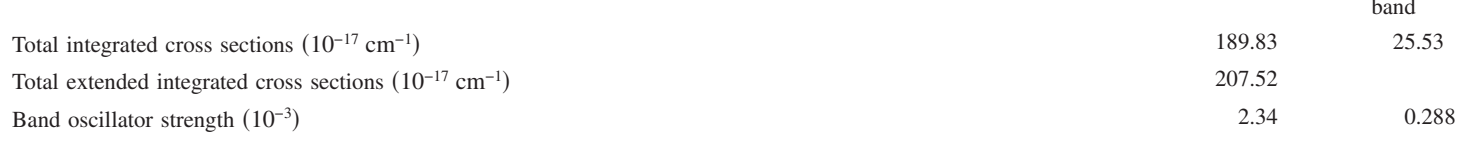

(b)

$\delta(1,0)$ band

\begin{tabular}{|c|c|c|c|c|c|c|c|c|c|}
\hline \multirow[b]{2}{*}{$J$} & \multicolumn{2}{|c|}{$R_{11}$} & \multicolumn{2}{|c|}{$P_{11}$} & \multirow[t]{2}{*}{$Q_{11}$} & \multicolumn{2}{|c|}{$R_{12}$} & \multicolumn{2}{|c|}{$P_{12}$} \\
\hline & $e e$ & $f f$ & $e e$ & $f f$ & & $e e$ & $f f$ & $e e$ & $f f$ \\
\hline 0.5 & \multicolumn{2}{|c|}{$2.01 \mathrm{~b}$} & & & \multicolumn{3}{|l|}{1.62} & & \\
\hline 1.5 & \multicolumn{2}{|c|}{3.78} & \multicolumn{2}{|c|}{$1.31 \mathrm{~b}$} & & \multicolumn{2}{|c|}{$1.14 \mathrm{~b}$} & & \\
\hline 2.5 & \multicolumn{2}{|c|}{4.95} & \multicolumn{2}{|c|}{3.25} & & \multicolumn{2}{|c|}{$1.90 \mathrm{~b}$} & \multicolumn{2}{|c|}{0.98} \\
\hline 3.5 & \multicolumn{2}{|c|}{$6.95 \mathrm{~b}$} & \multicolumn{2}{|c|}{4.45} & & \multicolumn{2}{|c|}{$3.31 \mathrm{~b}$} & \multicolumn{2}{|c|}{1.82} \\
\hline 4.5 & $6.43 \mathrm{~b}$ & 3.77 & \multicolumn{2}{|c|}{$6.08 \mathrm{~b}$} & & $2.61 \mathrm{~b}$ & $1.45 \mathrm{~b}$ & \multicolumn{2}{|c|}{2.19} \\
\hline 5.5 & 5.00 & $3.47 \mathrm{~b}$ & \multicolumn{2}{|c|}{$8.66 \mathrm{~b}$} & & $3.16 \mathrm{~b}$ & 1.28 & \multicolumn{2}{|c|}{2.50} \\
\hline 6.5 & $3.32 \mathrm{~b}$ & 3.90 & 3.65 & 2.83 & & 1.78 & $1.22 \mathrm{~b}$ & 1.29 & 0.93 \\
\hline 7.5 & 3.39 & 3.99 & $4.05 \mathrm{~b}$ & 3.20 & & $1.44 \mathrm{~b}$ & $2.42 \mathrm{~b}$ & 1.31 & 1.12 \\
\hline 8.5 & 4.01 & $4.96 \mathrm{~b}$ & $3.78 \mathrm{~b}$ & 2.58 & & 1.36 & 1.60 & 1.11 & 1.36 \\
\hline 9.5 & 3.43 & 4.32 & $3.36 \mathrm{~b}$ & $3.13 \mathrm{~b}$ & & 1.17 & 1.41 & $1.20 \mathrm{~b}$ & 1.03 \\
\hline 10.5 & 3.36 & 3.68 & $3.29 b$ & $2.98 \mathrm{~b}$ & & $1.14 \mathrm{~b}$ & 1.29 & $1.30 \mathrm{~b}$ & $1.04 \mathrm{~b}$ \\
\hline 11.5 & 3.12 & 3.55 & $2.99 \mathrm{~b}$ & $2.83 \mathrm{~b}$ & & 0.84 & $1.00 \mathrm{~b}$ & 1.23 & 1.22 \\
\hline 12.5 & 2.77 & 3.10 & $2.88 \mathrm{~b}$ & $2.75 \mathrm{~b}$ & & 0.85 & 0.79 & 1.30 & $0.94 \mathrm{~b}$ \\
\hline 13.5 & 2.55 & $3.28 \mathrm{~b}$ & 2.52 & $2.83 \mathrm{~b}$ & & 0.51 & 0.80 & $1.21 \mathrm{~b}$ & $0.88 \mathrm{~b}$ \\
\hline 14.5 & 2.26 & 2.75 & $2.38 \mathrm{~b}$ & $2.66 \mathrm{~b}$ & & $0.51 \mathrm{~b}$ & 0.62 & $0.98 \mathrm{~b}$ & $0.71 \mathrm{~b}$ \\
\hline 15.5 & 1.94 & 1.93 & $1.73 \mathrm{~b}$ & $2.15 \mathrm{~b}$ & & 0.44 & 0.28 & & \\
\hline 16.5 & 1.58 & 1.88 & $1.23 \mathrm{~b}$ & $1.36 \mathrm{~b}$ & & 0.76 & 0.10 & & \\
\hline 17.5 & 1.39 & $1.30 \mathrm{~b}$ & $0.98 \mathrm{~b}$ & 1.20 & & & & & \\
\hline 18.5 & 0.91 & $0.98 \mathrm{~b}$ & 0.52 & & & $0.36 \mathrm{~b}$ & & & \\
\hline & & & & & 1.62 & & & & \\
\hline nded & & & & & & & & & \\
\hline
\end{tabular}

$\delta(1,0)$ band

\begin{tabular}{|c|c|c|c|c|c|c|c|c|c|}
\hline & \multicolumn{2}{|c|}{$R_{22}$} & \multicolumn{2}{|c|}{$P_{22}$} & \multirow[t]{2}{*}{$Q_{22}$} & \multicolumn{2}{|c|}{$R_{21}$} & \multicolumn{2}{|c|}{$P_{21}$} \\
\hline$J$ & $e e$ & $f f$ & $e e$ & $f f$ & & $e e$ & $f f$ & $e e$ & $f f$ \\
\hline
\end{tabular}

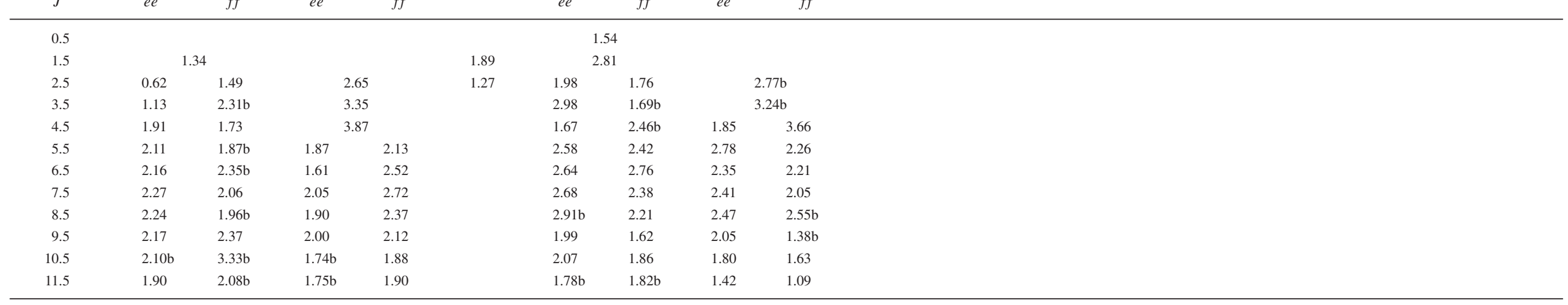




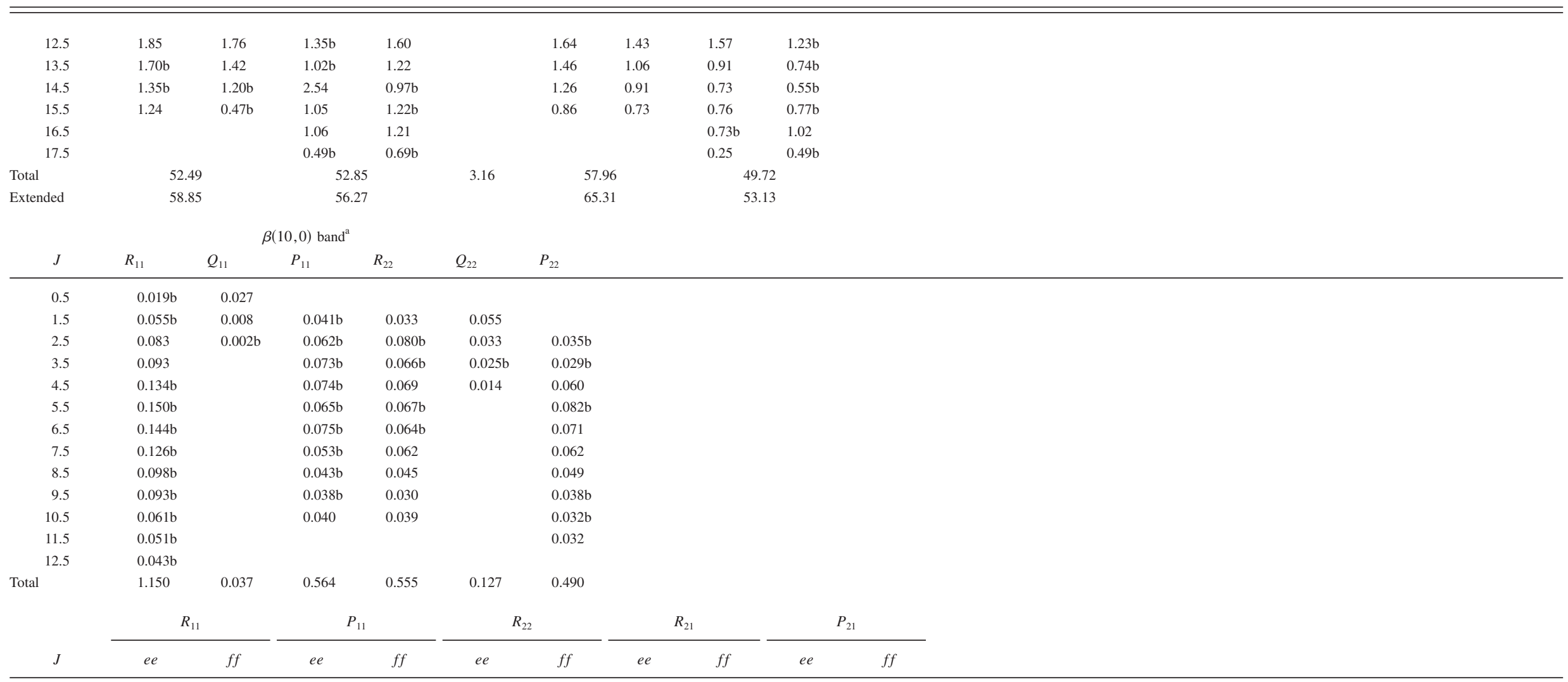

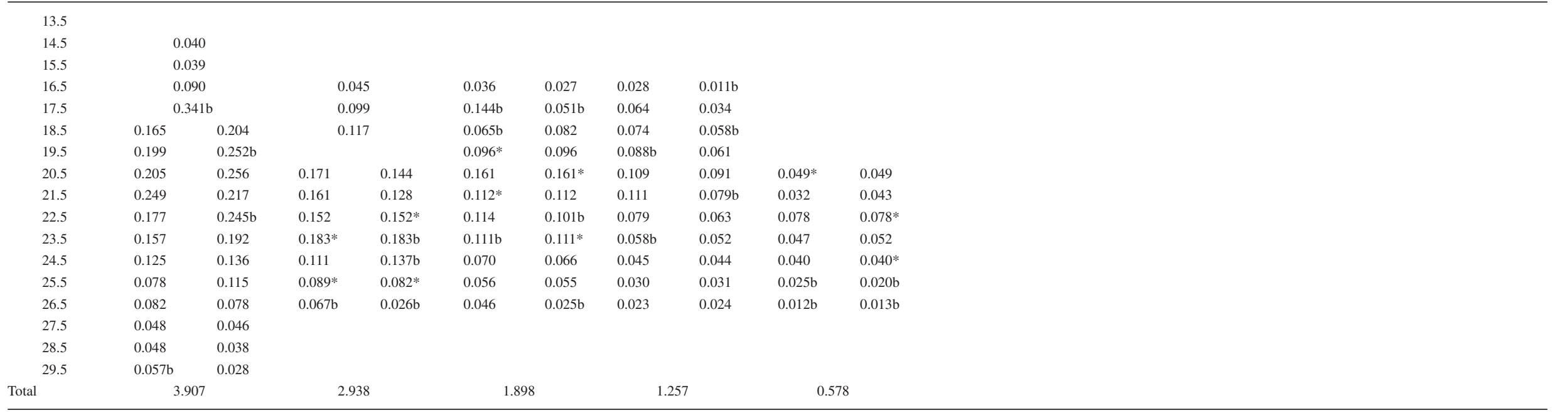




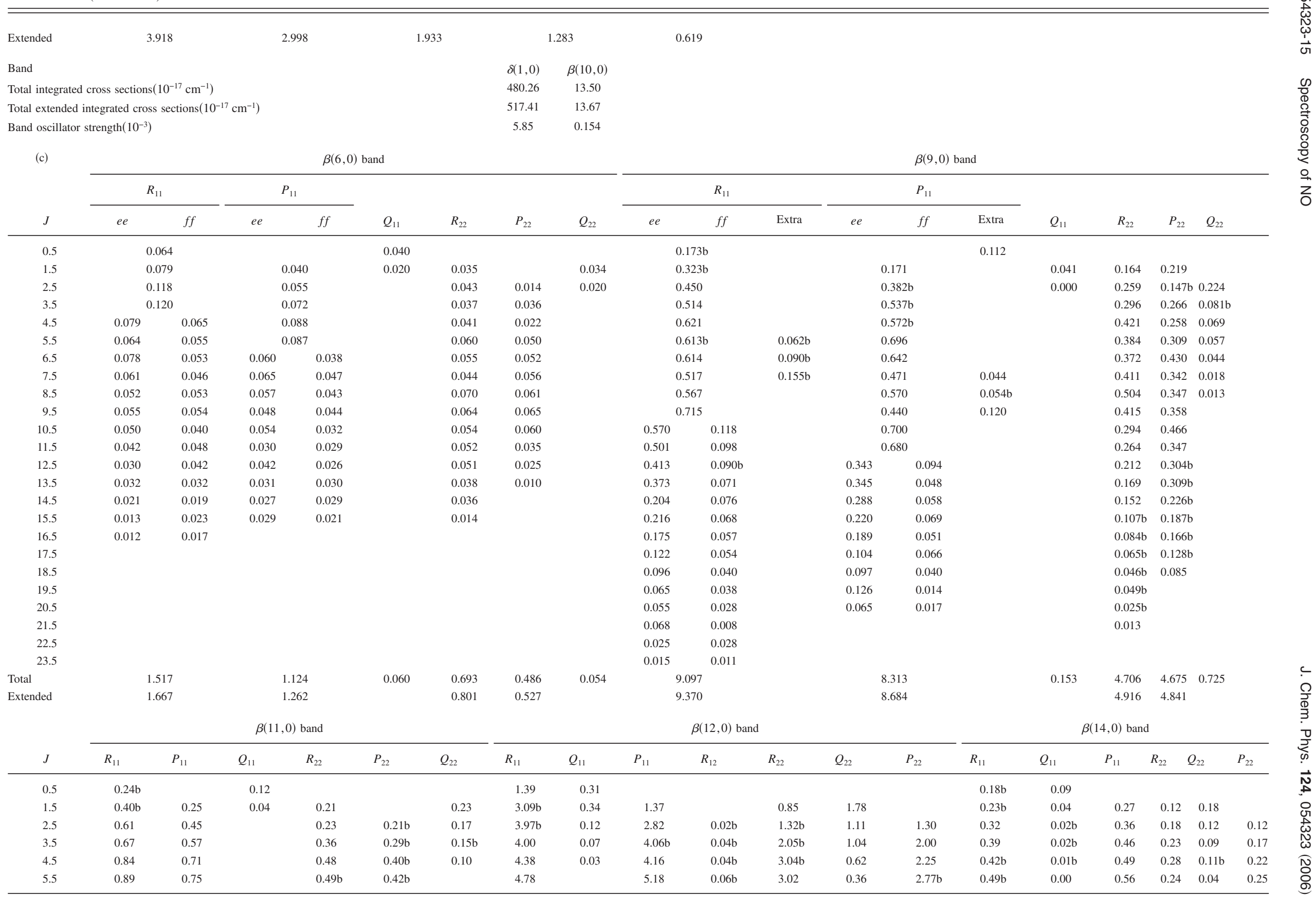




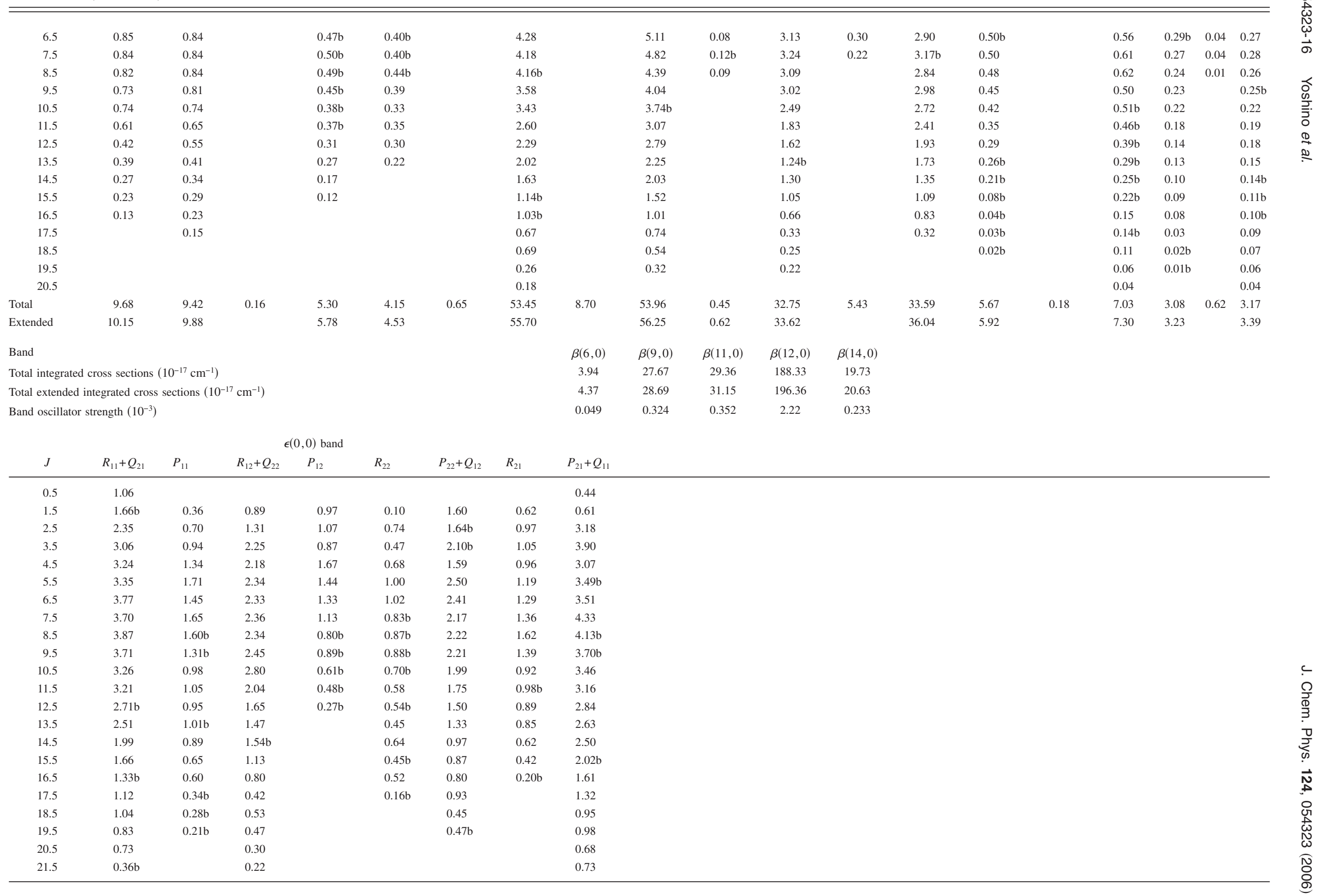




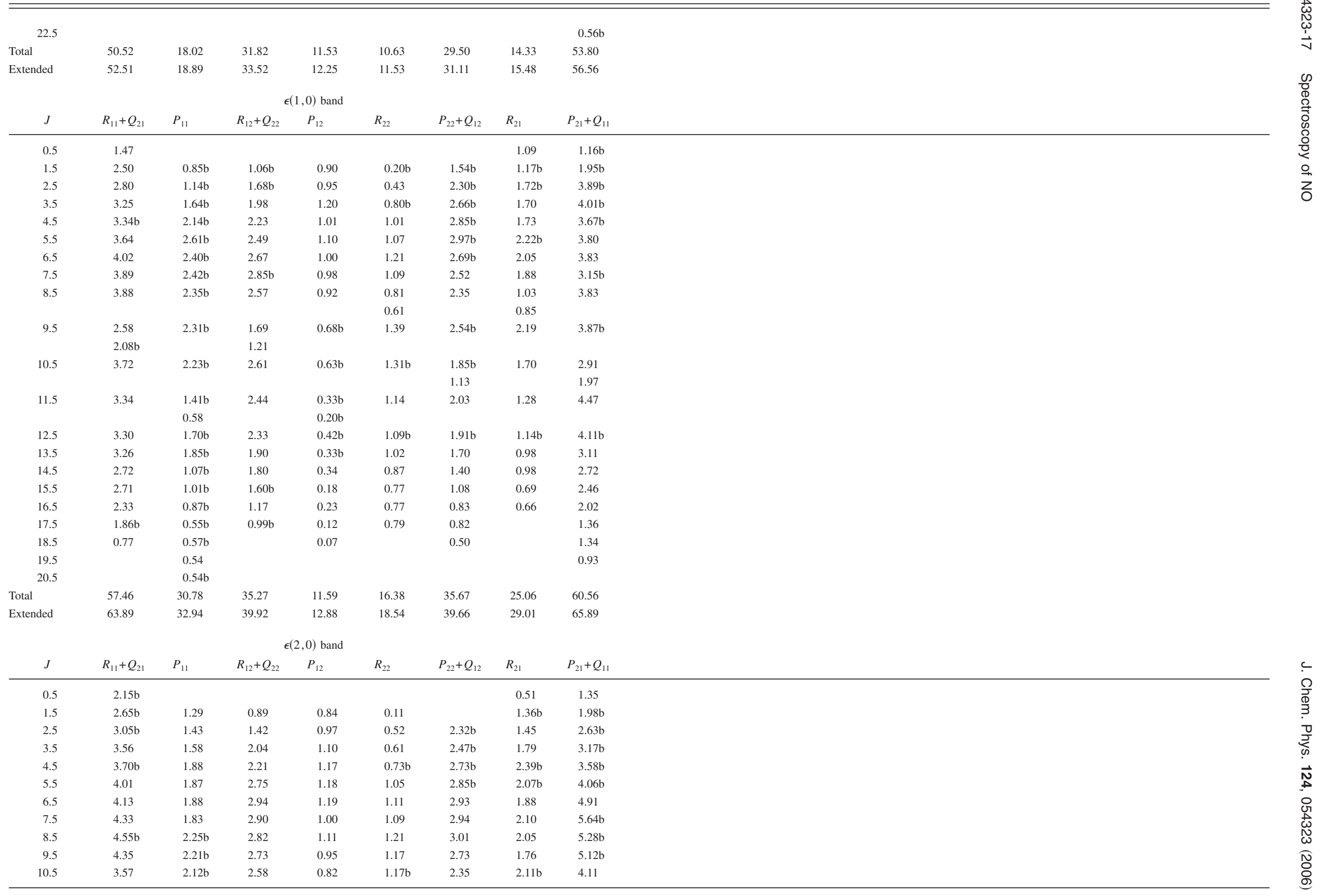




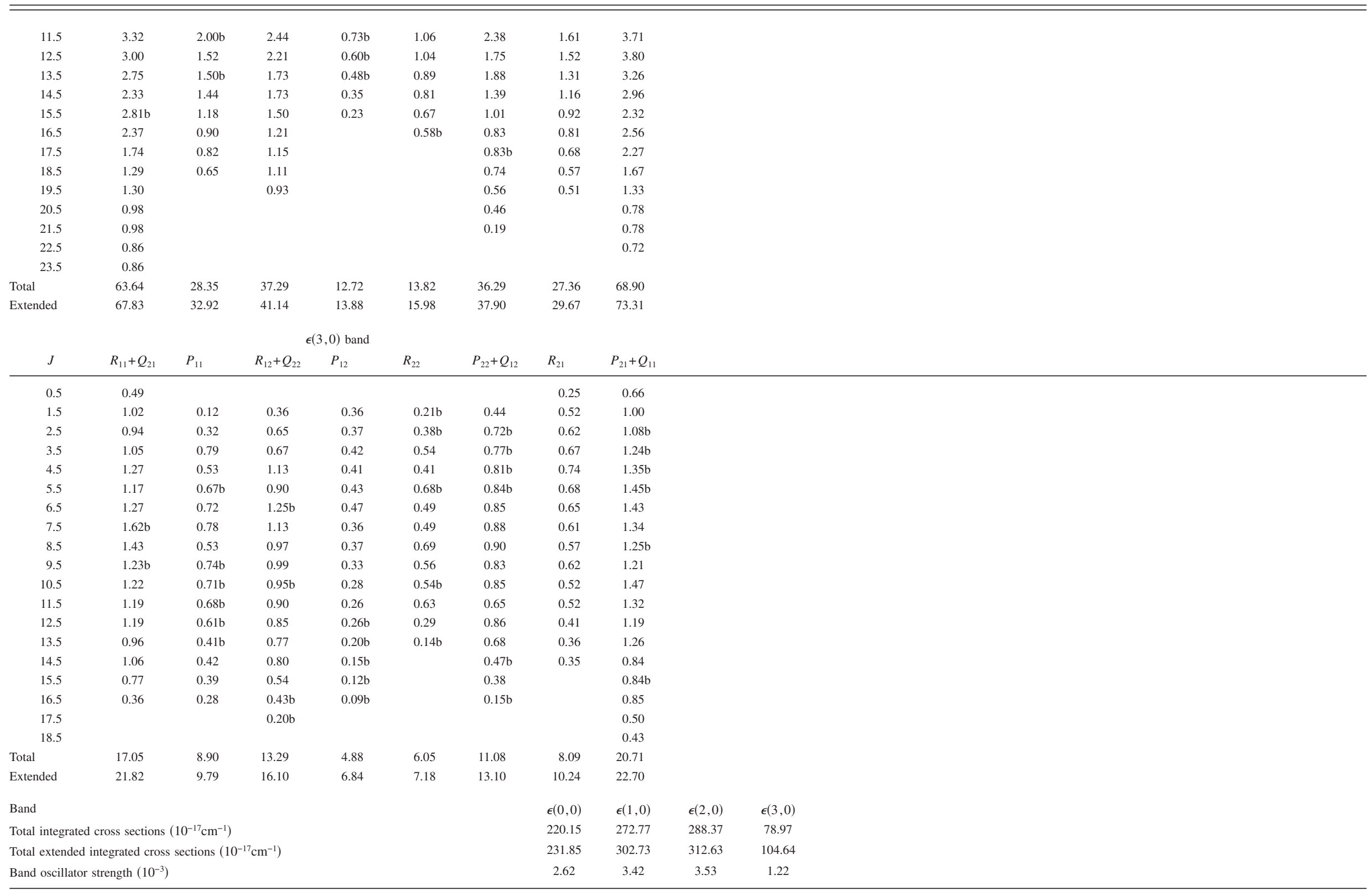




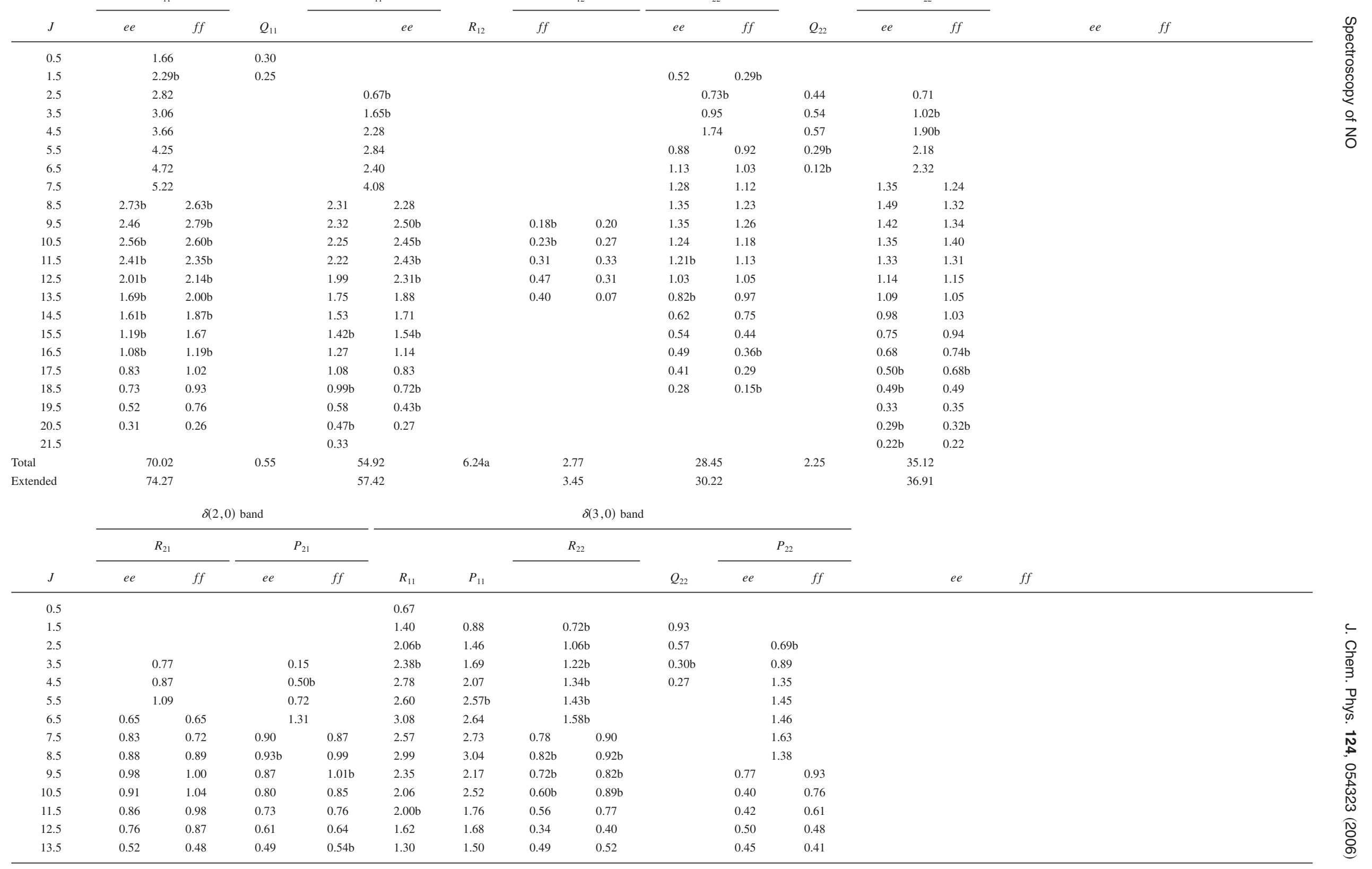




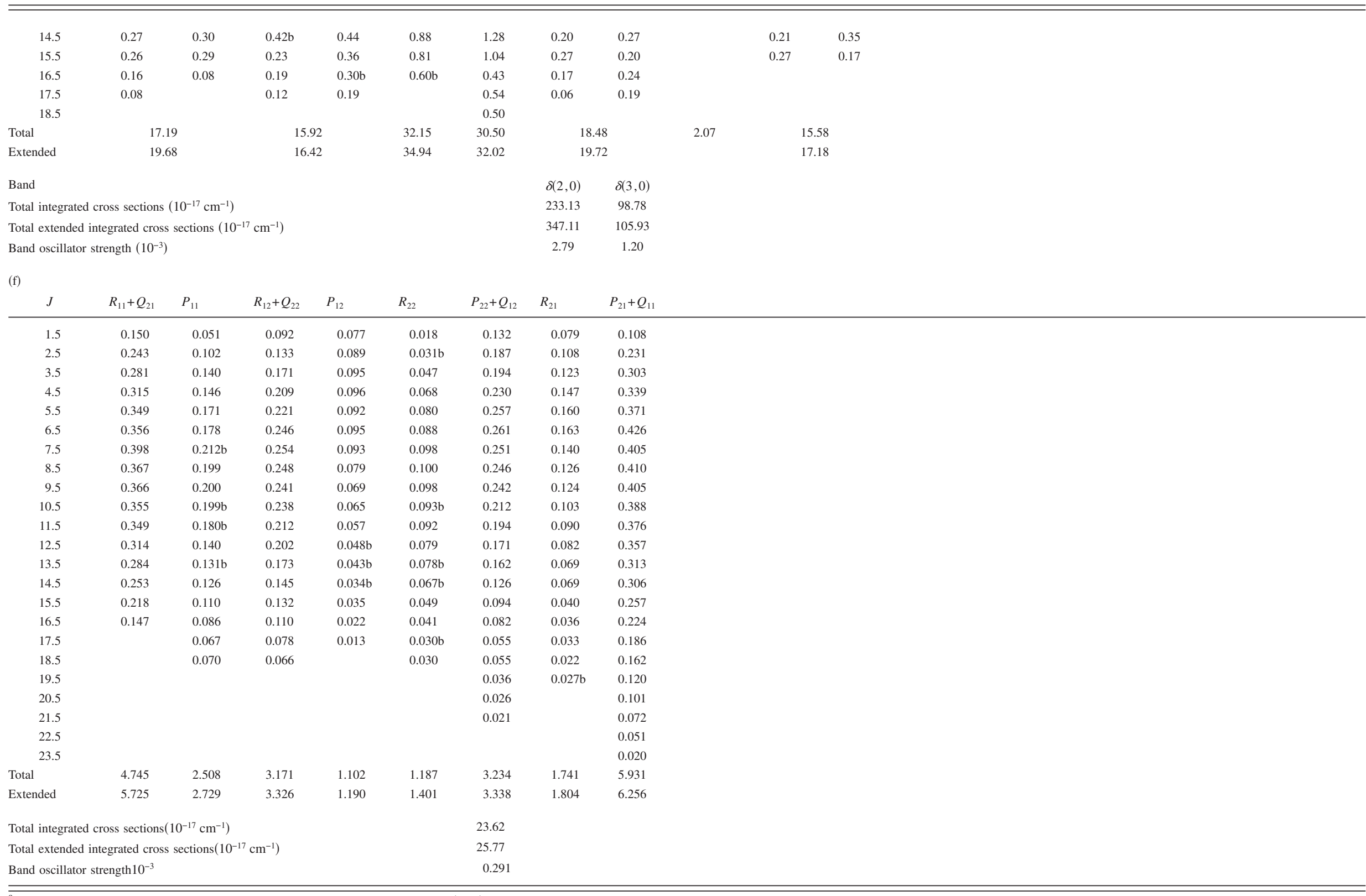

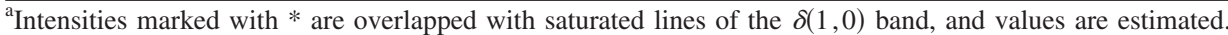




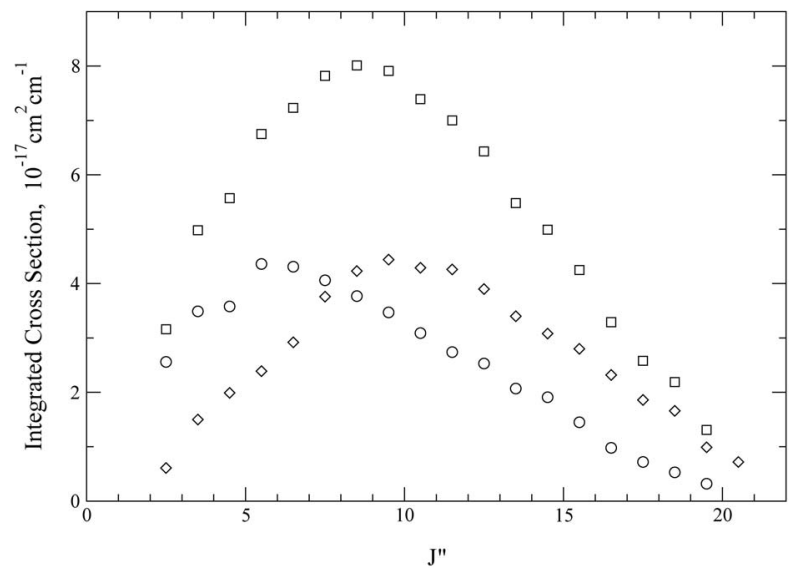

FIG. 2. Effects of the homogeneous perturbation on intensity distribution. $P_{11}$ branch lines of the $\delta(2,0)$ and $\beta(12,0)$ bands are presented by diamonds and circles, respectively. Sum of the both bands are given by squares. The branch lines do not follow by Boltzmann distribution, but the sum of them followed.

quantity is unity - and the integration of the cross section $\sigma(\nu)$ is performed over all of the rotational lines. The total integrated cross sections of observed lines for each branch are presented as "Total" in Table III. Observations of the rotational lines are mostly limited to $J \leqslant 20.5$. The effects from higher $J$ lines cannot be ignored. The contribution of these higher $J$ lines was obtained by extrapolating the cross sections up to $J=36.5$ by fitting the experimental points to a Boltzmann distribution curve, as shown in Fig. 4 of Ref. 6. The results of the extended contributions are also listed in Table III as "Extended." The total integrated cross sections and band oscillator strength of each band are presented at the bottom of each table.

The band oscillator strengths of the NO bands determined by the FTS measurements are compared with those of previous measurements in Table IV. The uncertainty in our measured band oscillator strength is estimated to be about $5 \%$, which includes uncertainties from line intensity measurements and extrapolation. The value under FTS/PB are our published values ${ }^{1-6}$ without any correction on the integrated cross sections. The value under FTS/CR are the results after intensity correction. The ratio of those values should not be constants, because the ratio depends on the observed optical depth. Our values, obtained by line-by-line integration, are all for single bands. In the cases of the $\delta(0,0)$ and $\delta(1,0)$ bands, the values for $\beta(7,0)$ and $\beta(10,0)$, respectively, should be added for comparison with other work.

Bethke ${ }^{14}$ measured integrated cross sections of pressurebroadened NO and gives a maximum error of $10 \%$ for his band oscillator strengths. His values agree well with ours, within the combined uncertainties, except for the $\epsilon(1,0)$ and $\gamma(3,0)$ bands. Cieslik ${ }^{28}$ obtained the band oscillator strength by two methods, extrapolation to zero pressure and use of the

TABLE IV. Band oscillator strength $\left(10^{-3}\right)$ of NO.

\begin{tabular}{|c|c|c|c|c|c|c|c|c|c|c|c|c|c|}
\hline \multirow[b]{2}{*}{ Band } & \multirow[t]{2}{*}{$\begin{array}{l}\text { Upper } \\
\text { level }\end{array}$} & \multicolumn{2}{|c|}{ FTS } & \multirow[b]{2}{*}{$\mathrm{LC}^{\mathrm{c}}$} & \multirow[b]{2}{*}{$\mathrm{CCB}^{\mathrm{d}}$} & \multirow[b]{2}{*}{$\mathrm{GL}^{\mathrm{e}}$} & \multicolumn{2}{|c|}{ Cieslik $^{\mathrm{f}}$} & \multirow[b]{2}{*}{ Beth. $^{\mathrm{g}}$} & \multirow[b]{2}{*}{$\mathrm{CP}^{\mathrm{h}}$} & \multirow[b]{2}{*}{ LGTM $^{\mathrm{i}}$} & \multirow[b]{2}{*}{$B E L^{j}$} & \multirow[b]{2}{*}{$\mathrm{MCY}^{\mathrm{k}}$} \\
\hline & & $\mathrm{CR}^{\mathrm{a}}$ & $\mathrm{PB}^{\mathrm{b}}$ & & & & B & $\mathrm{C}$ & & & & & \\
\hline$\gamma(3,0)$ & $A(3)$ & 0.291 & 0.269 & 0.30 & 0.356 & & 0.30 & 0.308 & 0.360 & & & & \\
\hline$\beta(6,0)$ & $B(6)$ & 0.049 & 0.048 & & 0.037 & & & & 0.0462 & & & & \\
\hline$\delta(0,0)$ & $C(0)$ & 2.34 & & & 2.29 & 3.52 & 2.5 & 2.84 & 2.49 & 5.6 & 2.4 & 2.0 & 2.2 \\
\hline$\beta(7,0)$ & $B(7)$ & 0.288 & & & 0.375 & & & & & & & & \\
\hline$\epsilon(0,0)$ & $D(0)$ & 2.62 & 2.47 & 1.87 & 2.63 & 2.51 & 2.6 & 2.53 & 2.54 & 2.54 & & & \\
\hline$\beta(9,0)$ & $B(9)$ & 0.324 & 0.265 & & 0.314 & & 0.37 & 0.356 & 0.358 & & & & \\
\hline$\delta(1,0)$ & $C(1)$ & 5.85 & 5.4 & & 6.01 & 8.53 & 6.0 & 5.63 & 5.78 & & & & \\
\hline$\beta(10,0)$ & $B(10)$ & 0.154 & & & & & & & & & & & \\
\hline$\epsilon(1,0)$ & $D(1)$ & 3.42 & 2.88 & 3.75 & 4.61 & 4.60 & 5.6 & 6.46 & 4.60 & & & & \\
\hline$\beta(11,0)$ & $B(11)$ & 0.352 & 0.344 & & 0.648 & & & & 0.362 & & & & \\
\hline$\beta(12,0)$ & $B(12)$ & 2.22 & & & 2.09 & 2.17 & & & 2.31 & & & & \\
\hline$\delta(2,0)$ & $C(2)$ & 2.79 & & & 3.08 & 2.94 & & & 2.74 & & & & \\
\hline$\epsilon(2,0)$ & $D(2)$ & 3.53 & & 3.16 & 3.67 & 3.16 & & & 3.32 & & & & \\
\hline$\beta(14,0)$ & $B(14)$ & 0.233 & & & 0.354 & & & & 0.201 & & & & \\
\hline$\delta(3,0)$ & $C(3)$ & 1.20 & & & 0.976 & & & & & & & & \\
\hline$\beta(15,0)$ & $B(15)$ & & & & 0.870 & & & & & & & & \\
\hline$\epsilon(3,0)$ & $D(3)$ & 1.22 & & 1.44 & 1.79 & & & & & & & & \\
\hline
\end{tabular}

${ }^{\mathrm{a}}$ Present works after intensity correction.

${ }^{\mathrm{b}}$ Our published works (Refs. 1-6).

${ }^{c}$ Luque and Crosley (1999) (Ref. 17).

${ }^{\mathrm{d}}$ Chan, Cooper, and Brion (1993) (Ref. 16).

${ }^{\mathrm{e}}$ Guest and Lee (1981) (Ref. 15).

${ }^{\text {f }}$ Cieslik (1977) (Ref. 28): (B) extrapolated to zero; (C) the curve of growth.

${ }^{\mathrm{g}}$ Bethke (1959) (Ref. 14).

${ }^{\mathrm{h}}$ Callear and Pilling (1970) (Ref. 29).

${ }^{\mathrm{i}}$ Lewis et al. (1989) (Ref. 30).

${ }^{\mathrm{j}}$ Brzozowski et al. (1976) (Ref. 31).

${ }^{\mathrm{k}}$ Mandelman et al. (1973) (Ref. 32). 
curve of growth, shown in Table IV under B and C, respectively. His values agree with ours except that his values for the $\epsilon(1,0)$ are larger. Guest and $\mathrm{Lee}^{15}$ measured integrated cross sections with a resolution of $0.03 \mathrm{~nm}$, but hold the optical density below 0.4 . They estimate uncertainty of $40 \%$ because the transition saturates even at low pressure. Chan et $a l .{ }^{16}$ observed the NO electronic spectra by using the dipole $(e, e)$ method of electron-impact excitation, which is free of optical instrumental function and saturation errors. However, their resolution $\left(0.048 \mathrm{eV}, 387 \mathrm{~cm}^{-1}\right.$, or $1.26 \mathrm{~nm}$ at $\left.180 \mathrm{~nm}\right)$ is not high enough to separate the band structures. They claimed the uncertainties of their measurements to be 5\%$10 \%$ for strong and partially resolved bands, with additional errors expected from the deconvolution. Their values agree reasonably well with ours except for the $\epsilon(1,0)$ band and the last four bands in Table IV, $\beta(14,0), \delta(3,0)$, and $\epsilon(3,0)$, together with $\beta(15,0)$, for which we did not make measurements. The large difference for the $\epsilon(3,0)$ band might be attributable to their deconvolution. The calculated values of Luque and Crosley ${ }^{17}$ are also included in Table IV, but they normalized to different lifetimes for the $\gamma$ band and the $\epsilon$ bands. Their values for the $\epsilon$ bands agree well with ours, including that for the $\epsilon(1,0)$ band, for which the values of the other measurements are much higher.

The line oscillator strengths could in principle be deduced from the band oscillator strengths using Hönl-London factors and the Boltzmann distribution. However, most of the upper levels of the NO bands are to some extent perturbed, homogeneously or heterogeneously. Consequently, as demonstrated in Fig. 2, the line intensities do not follow the Boltzmann distribution. This is especially the case for the strongly perturbed bands $\delta(2,0)$ and $\delta(3,0)$ and their partners. Therefore the actual measured integrated cross sections of the rotational lines become very important. The values presented here are for $295 \mathrm{~K}$, but they could be applied to any other temperature by dividing by the rotational population at $295 \mathrm{~K}$.

\section{SUMMARY}

This is the final report of the FTS/PF work. We have presented lists of the observed line positions and the term values for all bands observed except those published previously, ${ }^{1-7}$ together with their integrated cross sections. Line intensities for the bands in these previous publications have been corrected for instrumental effects, and the revised integrated cross sections are given here. The band oscillator strengths of all bands observed in this work are presented and compared with other published results. They are also provided after instrumental corrections.

\section{ACKNOWLEDGMENTS}

This work was supported in part by a NSF Division of Atmospheric Sciences Grant No. ATM-94-22854 to Harvard
College Observatory and by the NASA Upper Atmospheric Research Program under Grant No. NAG5-484 to the Smithsonian Astrophysical Observatory. We also acknowledge the Paul Instrument Fund of the Royal Society for the development of the vuv-FT spectrometer. The FTS measurements at the Photon Factory were made with the approval of the Photon Factory Advisory Committee (94G367). We thank Dr. Stark for the computation program for the correction of the line integrated cross sections. One of the authors (K.Y.) thanks the Japan Society for the Promotion of Science for support. Another author (A.P.T.) thanks NATO for its Grant for international collaboration in research (890224)

${ }^{1}$ K. Yoshino, J. R. Esmond, W. H. Parkinson et al., J. Chem. Phys. 109, 1751 (1998)

${ }^{2}$ T. Imajo, K. Yoshino, J. R. Esmond et al., J. Chem. Phys. 112, 2251 (2000).

${ }^{3}$ J. Rufus, K. Yoshino, J. R. Esmond, A. P. Thorne, T. Imajo, K. Ito, and T. Matsui, J. Chem. Phys. 115, 3719 (2001).

${ }^{4}$ A. S.-C. Cheung, D. H.-Y. Lo, K. W.-S. Leung, K. Yoshino, A. P. Thorne, J. E. Murray, K. Ito, T. Matsui, and T. Imajo, J. Chem. Phys. 116, 155 (2002).

${ }^{5}$ J. Rufus, K. Yoshino, A. P. Thorne, J. E. Murray, T. Imajo, K. Ito, and T. Matsui, J. Chem. Phys. 117, 10621 (2002).

${ }^{6}$ A. S.-C. Cheung, A. L. Wong, D. H.-Y. Lo, K. W.-S. Leung, K. Yoshino, A. P. Thorne, J. E. Murray, K. Ito, T. Matsui, and T. Imajo, J. Chem. Phys. 119, 8373 (2003)

${ }^{7}$ A. P. Thorne, J. Rufus, K. Yoshino, A. S.-C Cheung, and T. Imajo, J. Chem. Phys. 122, 179901 (2005).

${ }^{8}$ J. E. Murray, K. Yoshino, J. R. Esmond, W. H. Parkinson, Y. Sun, A. Dalgarno, A. P. Thorne, and G. Cox, J. Chem. Phys. 101, 62 (1994).

${ }^{9}$ G. Herzberg, A. Lagerqvist, and E. Miescher, Can. J. Phys. 34, 622 (1956).

${ }^{10}$ A. Lagerqvist and E. Miescher, Helv. Phys. Acta 31, 221 (1958).

${ }^{11}$ R. Gallusser and K. Dressler, J. Chem. Phys. 76, 4311 (1982).

${ }^{12}$ M. Raoult, J. Chem. Phys. 87, 4736 (1987).

${ }^{13}$ V. D. Braun, K. P. Huber, and M. Verloet, J. Mol. Spectrosc. 203, 65 (2000).

${ }^{14}$ G. W. Bethke, J. Chem. Phys. 31, 662 (1959).

${ }^{15}$ J. A. Guest and L. C. Lee, J. Phys. B 14, 3401 (1981).

${ }^{16}$ W. F. Chan, G. Cooper, and C. E. Brion, Chem. Phys. 170, 111 (1993).

${ }^{17}$ J. Luque and D. R. Crosley, J. Phys. Chem. 111, 7405 (1999).

${ }^{18}$ E. Mayor, A. M. Velasco, and I. Martin, J. Chem. Phys. 123, 114305 (2005).

${ }^{19}$ J. W. Brault (private communication).

${ }^{20}$ P. M. Dooley, B. R. Lewis, S. T. Gibson et al., J. Chem. Phys. 109, 3856 (1998).

${ }^{21}$ R. D. Hudson and V. L. Carter, J. Opt. Soc. Am. 58, 227 (1968).

${ }^{22}$ G. Stark, K. Yoshino, P. L. Smith, K. Ito, and W. H. Parkinson, Astrophys. J. 369, 574 (1991).

${ }^{23} \mathrm{H}$. Lefebvre-Brion and R. W. Field, The Spectra and Dynamics of Diatomic Molecules (Elsevier, San Diego, 2004).

${ }^{24}$ E. Miescher (private communication).

${ }^{25}$ C. Amiot, R. Bacis, and G. Guelachvili, Can. J. Phys. 56, 251 (1978).

${ }^{26}$ Ch. Jungen and E. Miescher, Can. J. Phys. 46, 987 (1968).

${ }^{27}$ R. Engleman, Jr. and P. E. Rouse, J. Mol. Spectrosc. 37, 240 (1971).

${ }^{28}$ S. Cieslik, Ci. Sci. Acad. Roy. Belg. 63, 884 (1977).

${ }^{29}$ A. B. Callear and M. J. Pilling, Trans. Faraday Soc. 66, 1886 (1970).

${ }^{30}$ B. R. Lewis, S. T. Gibson, L. W. Torop, and D. G. McCoy, Geophys. Res. Lett. 25, 2457 (1998).

${ }^{31}$ J. Brzozowski, P. Erman, and M. Lyyra, Phys. Scr. 14, 290 (1976).

${ }^{32}$ M. Mandelman, T. Carrington, and R. Young, J. Chem. Phys. 58, 84 (1973). 